\title{
CONCERNING CERTAIN TOPOLOGICALLY FLAT SPACES $\dagger$
}

\author{
BY \\ F. BURTON JONES
}

In any space satisfying Axioms 0, 1, 2, 3, 4, and 5 of R. L. Moore's Foundations of Point Set Theory $\ddagger$ a large body of Plane Analysis Situs theorems holds true. Nevertheless, Moore has an example§ of a completely separable space satisfying these axioms which because of its "hilly" character is not homeomorphic with a subset of a plane or a sphere. In this paper Axiom 5 is replaced by an axiom, Axiom $5_{1}^{*}$, which is not satisfied by spaces of this "hilly" character. As a result, any completely separable (or metric) space satisfying Axioms $0-4$ and $5{ }_{1}{ }^{*}$ is homeomorphic with a subset of a plane or a sphere. However, in the presence of Axioms 0-4 neither of the Axioms 5 and $5_{1}^{*}$ follows from the other.

Axiom $5_{1} \|$ of Foundations suggests the following two axioms:

Aхгом $51^{1}$. If $P$ is a point of region $R$, there exists in $R$ a domain $D$ containing $P$ such that the boundary of $D$ is compact. $\Uparrow$

Aхгом $5{ }_{1}^{2}$. If $P$ is a point of a region $R$, there exists in $R$ a domain $D$ containing $P$ such that the boundary of $D$ is connected. $\dagger \dagger$

There is, however, a certain amount of similarity between these last two axioms. For suppose that a space satisfies Moore's Axioms 0, 1, and 2 and

† Parts of this paper were presented to the Society on October 28, 1933, June 20, 1934, and February 23, 1935; received by the editors July 27, 1936. The author wishes to acknowledge his indebtedness and appreciation to Professor R. L. Moore for much encouragement and many helpful suggestions during this investigation and for having stimulated his interest in mathematics.

$\ddagger$ American Mathematical Society Colloquium Publications, volume XIII, New York, 1932. Hereafter, in this paper, this book will be referred to as Foundations.

$\S$ This example of Moore's, although unpublished, has been well known among his students for some four or five years.

$\|$ If $P$ is a point of a region $R$, there exists a connected domain $D$ containing $P$ and bounded by a compact continuum $T$ such that $D+T$ is a subset of $R$.

I A space satisfying Axiom $5_{1}{ }^{1}$ is said to be locally peripherally compact.

i† A space satisfying Axiom $5_{1}^{2}$ is said to be locally peripherally connected. 
Axiom $5_{1}^{1} \cdot \dagger$ Then if $P$ is a point of a region $R$, there exists a region $R^{\prime}$ containing $P$ and lying together with its boundary in $R$. And by Axiom $5_{1}^{1}$ there exists in $R^{\prime}$ a domain $D^{\prime}$ containing $P$ whose boundary $\beta$ is compact. But $\beta$ may be covered by a finite collection $D_{1}, D_{2}, D_{3}, \cdots, D_{j}$ of connected domains such that for each $n, n \leqq j, \bar{D}_{n}$ is a subset of $R-P$. $\ddagger$ Let $D$ denote the component of $R-\sum \bar{D}_{n}$ which contains $P$. Thus the following theorem holds true and will be used hereafter as

Aхіом $5_{1}^{*}$. If $P$ is a point of a region $R$, there exists in $R$ a domain $D$ containing $P$ whose boundary is a subset of the sum of a finite number of continua lying in $R-D$.

In a sense Axiom $5_{1}{ }^{*}$ is common to both Axioms $5_{1}{ }^{1}$ and $5_{1}{ }^{2}$ in that it follows as a theorem from either of them in the presence of Axioms 0, 1, and 2.

Now let $S$ denote a space satisfying Moore's Axioms 0, 1, and 2 .

Theorem A. If Axiom $5_{1}^{*}$ holds true at each of two distinct points $A$ and $B$ of $S$ and no point of $S$ separates $A$ from $B$, then there exists in $S$ a simple closed curve $J$ containing $A$ and $B . \S$

Proof. Let $A B$ denote an arc from $A$ to $B$. If $R$ is a region containing $A$ but not $B$, suppose that there exists no point $O$ of $A B \cdot R$ such that no point $X$ of the interval $A O$ of $A B$ separates $A$ from $O$ in $R$. Then there exists a sequence $\alpha$ of points of $A B$ which converge to $A$ such that if $X$ and $Y$ are points of $\alpha$ and $X$ follows $Y$ in $\alpha$, then $X$ separates $A$ from $Y$ in $R$. Hence, for each point $X$ of $\alpha$ except the first, $R-X$ is the sum of two domains $U_{X}$ and $V_{X}$ such that $V_{X}$ contains $A$ and $U_{X}$ contains every point of $\alpha$ preceding $X$ in $\alpha$. But no point separates $A$ from $B$; so for each point $X$ of $\alpha$, there exists

$\dagger$ Axioms referred to are stated either in the text of this paper or in Foundations.

Aхгом 0 . Every region is a point set.

Axiom 1. There exists a sequence $G_{1}, G_{2}, G_{3}, \cdots$ such that (1) for each $n, G_{n}$ is a collection of regions covering $S$; (2) for each $n, G_{n+1}$ is a subcollection of $G_{n}$; (3) if $R$ is any region whatsoever, $X$ is a point of $R$, and $Y$ is a point of $R$ either identical with $X$ or not, then there exists a natural number $m$ such that if $g$ is any region belonging to the collection $G_{m}$ and containing $X$ then $\bar{g}$ is a subset of $(R-Y)+X$; (4) if $M_{1}, M_{2}, M_{3}, \cdots$ is a sequence of closed point sets such that, for each $n, M_{n}$ contains $M_{n+1}$ and, for each $n$, there exists a region $g_{n}$ of the collection $G_{n}$ such that $M_{n}$ is a subset of $\bar{g}_{n}$, then there is at least one point common to all the point sets of the sequence $M_{1}, M_{2}, M_{3}, \cdots$.

Axıом 2. If $P$ is a point of a region $R$ there exists a non-degenerate connected domain containing $P$ and lying wholly in $R$.

$\ddagger$ If $M$ denotes a point set, $\bar{M}$ denotes the point set consisting of $M$ and all the limit points of $M$.

$\S$ See Theorem 40 of Chapter II of Foundations and G. T. Whyburn, On the cyclic connectivity theorem, Bulletin of the American Mathematical Society, vol. 37 (1931), pp. 429-433.

|| Theorem 1 of Chapter II of Foundations: If $A$ and $B$ are distinct points of a connected domain D, there exists a simple continuous arc from $A$ to $B$ that lies wholly in $D$. 
an arc from $A$ to $B$ lying in $S-X$, and in this arc there exists a segment $\overparen{P E}$ lying in $R$ such that $A B \cdot P E=P$ and $E$ belongs to the boundary of $R$. $\dagger$ Therefore, if for no $X$ of $\alpha$ is $P$ identical with $A$, there exist a subsequence $X_{1}, X_{2}, X_{3}, \cdots$ of $\alpha$ and a sequence $P_{1} E_{1}, P_{2} E_{2}, P_{3} E_{3}, \cdots$ of arcs such that for each $n$ (1) $A B \cdot P_{n} E_{n}=P_{n}$, (2) $E_{n}$ belongs to the boundary of $R$, (3) $\widetilde{P_{n} E_{n}}$ is a subset of $R$, and (4) $X_{n+1}$ separates $\sum_{i=1}^{n} \widetilde{P_{i} E_{i}}$ from $A$ in $R$. But (1), (2), and (3) lead to a contradiction of (4), for since there exists in $R$ a domain $D$ containing $A$ whose boundary is a subset of the sum of a finite collection $\Delta$ of continua lying in $R-D$, there exists a continuum $H$ of $\Delta$ lying in $R-A$ such that $H$ does not contain more than a finite number of the points $X_{1}, X_{2}, X_{3}, \cdots$ but for infinitely many values of $n, H$ contains a point of $P_{n} E_{n}$. Therefore, either there exists an arc $P E$ which is a subset of an arc from $A$ to $B$ such that $P E \cdot A B=P=A$, or if $R$ is a region containing $A$ but not $B$, there exists a point $O$ of $A B \cdot R$ such that no point of the interval $A O$ of $A B$ separates $A$ from $O$ in $R$.

Suppose the latter is true. Let $R_{1}, R_{2}, R_{3}, \cdots$ be a monotonic sequence of regions closing down on $A$ and $O_{1}, O_{2}, O_{3}, \cdots$ be a sequence of points of $A B$ such that, for each $n$, (1) the interval $A O_{n}$ of $A B$ is a subset of $R_{n}$, (2) no point $X$ of $A O_{n}$ separates $A$ from $O_{n}$ in $R_{n}$. For each $n$ and each point $X$ of $A O_{n}$, there exists an arc $T_{x n}$ lying in $R_{n}$ and having only its end points in common with $A B$ such that the segment $S_{x n}$ of $A B$ between these end points contains $X$ and is a subset of $R_{n}$. For each $n$, there exists a finite collection of the segments $S_{x n}$ covering the $\operatorname{arc} O_{n+1} O_{n+2}$ of $A B$. But each $S_{x n}+T_{x n}$ is a simple closed curve lying in $R_{n}$. Hence there exists a sequence $J_{1}, J_{2}, J_{3}, \cdots$ of simple closed curves such that (1) for each $n, J_{n}$ and $J_{n+1}$ have at least two points in common, and (2) if $R$ is a region containing $A$ there exists a number $\delta$ such that if $n>\delta, J_{n}$ is a subset of $R$. By Theorem 35 of Chapter II of Foundations there exists in $\sum J_{n}$ a simple closed curve $J_{A}$ containing $A$ and a segment of $A B$.

If, on the other hand, there exists an $\operatorname{arc} P E$ which is a subset of an arc $P B$ from $A$ to $B$ such that $P E \cdot A B=P=A$, then there exists in $P B$ an arc $P X_{1}$ such that $P$ and $X_{1}$ are the only points $P X_{1}$ has in common with $A B$. But $P X_{1}+A X_{1}$ (of $A B$ ) forms a simple closed curve containing $A$ and some segment of $A B$.

So in either case there exists a simple closed curve $J_{A}$ containing $A$ and a segment $\overparen{O F}$ of $A B$. Likewise, there exists a simple closed curve $J_{B}$ containing $B$ and a segment $\overparen{Y E}$ of $A B$. Now for each point $X$ of the interval $F Y$ of $A B$, there exists an arc $T_{x}$ such that $T_{x}$ has only its end points in common with $A B$, and the segment $S_{x}$ of $A B$ lying between these end points contains $X$.

$\dagger$ If $A B$ is an arc with end points $A$ and $B, \overparen{A B}$ is used to denote $A B-(A+B)$. 
Some finite collection of these segments $S_{x}$ covers the arc $F Y$. Since every $S_{x}+T_{x}$ is a simple closed curve, there exists a sequence of simple closed curves $J_{1}=J_{A}, J_{2}, \cdots, J_{k}=J_{B}$ such that for each $n, n<k, J_{n}$ contains at least two points of $J_{n+1}$. By Theorem 35 of Chapter II of Foundations there exists in the sum of these simple closed curves a simple closed curve $J$ containing $A$ and $B . \dagger$

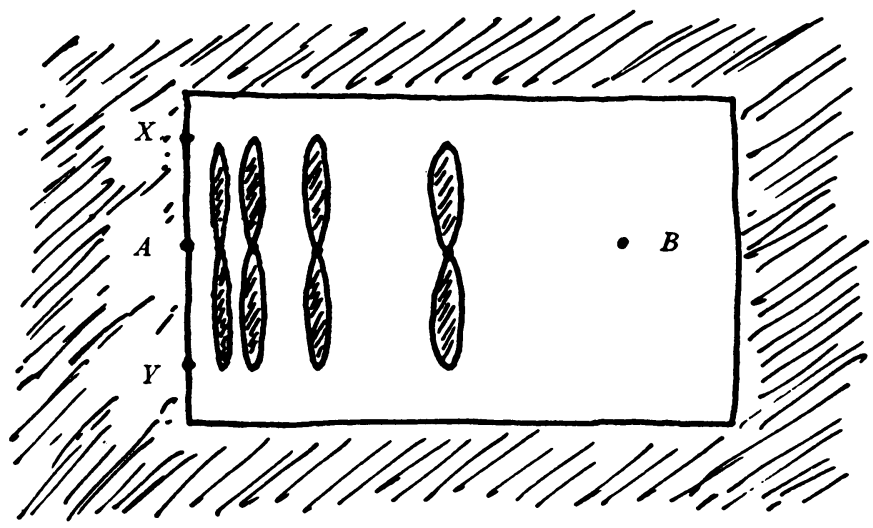

FIGURE 1

The example indicated in Figure 1 is a subset of a plane with the shaded portions removed. Except for those points of the arc $X A Y$ different from $A$, the boundary points of these shaded portions are not removed. It will be easily seen that the result is a connected, connected im kleinen inner limiting subset of the plane and hence satisfies Axioms 0,1 , and 2. Also Axiom $5_{1}^{*}$ is satisfied at every point except $A$. As a matter of fact, the space is locally compact at every point except $A$. It is also true in this space that if $P$ is a point and $R$ is a region containing $A$, there exists in $R$ an arc (or a simple closed curve) separating $A$ from $P$. The space contains no cut points. Yet there exists no simple closed curve containing $A$ and $B$.

$\dagger$ This argument may be slightly modified to establish a proposition somewhat more general than Theorem A.

THEOREM A'. If (1) $S$ is a space satisfying $R$. L. Moore's Axioms 0, 1, and 2, (2) neither of the distinct points $A$ and $B$ of $S$ is a cut point nor a local end point of $S$, and (3) no point separates $A$ from $B$ in $S$, then $A$ and $B$ lie on a simple closed curve in $S$. (See G. T. Whyburn, The cyclic and higher connectivity of locally connected spaces, American Journal of Mathematics, vol. 53 (1931), pp. 427-442.)

The modification may be outlined as follows: In the first paragraph of the proof, if $A$ is not a local end point, $\prod V_{X}$ contains a point $E^{\prime}$ distinct from $A$ which belongs to the component of $R$ that contains $A$. Let $A E^{\prime}$ denote an arc from $A$ to $E^{\prime}$ in $R$ and let $E^{\prime} B$ denote an arc from $E^{\prime}$ to $B$ in $S-A$. Then $A E^{\prime}+E^{\prime} B$ contains an arc $P E$ which is a subset of an arc from $A$ to $B$ such that $P E \cdot A B=P=A$. This takes us up to the last sentence of the first paragraph of the proof, which remains unchanged from there on. 
Suppose now that, in addition to Axioms 0-2, $S$ satisfies R. L. Moore's Axiom $4 \nmid$ and Axiom 51*

TheOREM B. The space $S$ is either acyclic or contains no cut point.

Proof. Suppose that $S$ contains a simple closed curve $J$. Then it is evident from Axiom 4 that $S$ is connected. Suppose that some point $X$ separates $S$. The point $X$ does not belong to $J$, for it is clear from Axiom 4 that no point of a simple closed curve separates $S$. Let $A X$ denote an arc with end points $A$ and $X$ such that $A X \cdot J=A$. Let $M$ denote the set of cut points of $S$ that belong to $A X$ and let $B$ denote the first point of $\bar{M}$ in the order from $A$ to $X$. The point $B$ is a cut point of $S$. For if it is not, then $B$ is a sequential limit point of a sequence $B_{1}, B_{2}, B_{3}, \cdots$ of $M$ and at the same time lies on a simple closed curve $C$ containing a segment of $A X$ which contains $B$; hence, $C$ contains points of the sequence $B_{1}, B_{2}, B_{3}, \cdots$ which contradicts Axiom 4. But since the segment $A B$ of $A X$ contains no point of $M$, no point separates $A$ from $B$ in $X$ and by Theorem A there exists a simple closed curve containing $A$ and $B$ which again contradicts Axiom 4 .

It is not the purpose of this paper to treat the acyclic case; $;$ so we shall at this point assume R. L. Moore's Axiom 3.§

\section{Consequences of Axioms $0-4$ and $5_{1}^{*}$.}

ThEOREM 1. If $A$ and $B$ are distinct points, there exists a simple closed curve containing $A$ and $B$.

THEOREM 2. If $A$ and $B$ are distinct points, there exists a simple closed curve separating $A$ from $B$.

Proof. There exists a simple closed curve $J$ such that $J$ is the sum of two $\operatorname{arcs} A X B$ and $A Y B$. Since $S-J$ is the sum of two connected domains $I$ and $E$ each having $J$ for its boundary, there exist two arcs $X_{1} O_{1} Y_{1}$ and $X_{2} O_{2} Y_{2}$ such that (1) the points $X_{1}$ and $X_{2}$ lie on the segment $A X B$ and the points $Y_{1}$ and $Y_{2}$ lie on the segment $A Y B$, and (2) the segment $X_{1} O_{1} Y_{1}$ is a subset of $I$ and the segment $X_{2} \mathrm{O}_{2} Y_{2}$ is a subset of $E$. The simple closed curve formed by the sum of the four arcs $X_{1} O_{1} Y_{1}, X_{2} O_{2} Y_{2}, X_{1} X_{2}$ (of $\left.A X B\right)$, and $Y_{1} Y_{2}$ (of $A Y B$ ) separates $A$ from $B$ in $S$.

† If $J$ is a simple closed curve, $S-J$ is the sum of two mutually separated connected point sets such that $J$ is the boundary of each of them.

$\ddagger$ Such a space if connected would be a Regular (Menger) Curve, and these curves have been studied considerably already. See Foundations for the definition and references on Regular (Menger) Curves.

$\S$ If $O$ is a point, $S-O$ is connected. 
THEOREM 3. If $P$ is a point not belonging to the closed and compact point set $H$, there exists a simple closed curve separating $P$ from $H$.

Proof. For each point $X$ of $H$ there exists a simple closed curve separating $P$ from $X$. Since $H$ is closed and compact, there exists a finite collection of simple closed curves $J_{1}, J_{2}, \cdots, J_{n}$ such that if $X$ is any point of $H$ some one of them separates $P$ from $X$. For each integer $i, i \leqq n$, let $T_{i}$ denote an arc from $J_{1}$ to $J_{i}$ lying in $S-P$ and let $M$ denote the compact continuum $J_{1}+J_{2}+\cdots+J_{n}+T_{1}+T_{2}+\cdots+T_{n}$. The continuum $M$ separates $P$ from $H-H \cdot M$. Now for each point $X$ of $M$ there is a simple closed curve separating $X$ from $P$. Let $C_{1}, C_{2}, \cdots, C_{j}$ denote a finite collection of these whose interiors with respect to $P$ as the point at infinity $\dagger$ cover $M$. By Theorem 13 of Chapter III of Foundations, $C_{1}+C_{2}+\cdots+C_{j}$ contains a simple closed curve separating $P$ from $M$ and hence, separating $P$ from $H$.

With the help of Theorem 3, the arguments of R. L. Moore with slight modifications prove that a number of the theorems of Chapter IV of Foundations hold true in this space. Of these the following five theorems will be used.

Theorem 4. If the points $A$ and $B$ belong to different components of the closed and compact point set $M$, there exists a simple closed curve separating $A$ from $B$ and containing no point of $M$ (Theorem 10 of Chapter IV of Foundations).

TheOREM 5. If $\alpha$ and $\beta$ are two connected point sets and neither of the two mutually exclusive closed and compact point sets $H$ and $K$ separates $\alpha$ from $\beta$, then $H+K$ does not separate $\alpha$ from $\beta$ (Theorem 16).

THEOREM 6. If the common part of the closed and compact point sets $H$ and $K$ is a continuum and neither $H$ nor $K$ separates the point $A$ from the point $B$, then $H+K$ does not separate $A$ from $B$ (Theorem 18 ).

TheOREм 7. No arc separates $S$ (Theorem 19).

THEOREM 8. If the points $A$ and $B$ are separated from each other by the closed and compact point set $M$ then they are separated from each other by a continuum which is a subset of $M$ and which contains no proper subset that separates $A$ from $B$ (Theorem 24).

THEOREM 9. If the compact continuum $K$ does not separate the point $A$ from the point $B$ and $G$ is a finite collection of compact continua such that (1) the common part of any two elements of $G$ is a subset of $K$, and (2) if $H$ is any element of $G, H$ does not separate $A$ from $B$ and $H \cdot K$ is either connected or vacuous, then $K+G^{*}$ does not separate $A$ from $B$.

$\dagger$ For the definition of certain terms and phrases used without definition in this paper the reader is referred to Foundations. 
Theorem 9 may be established by a finite number of applications of Theorems 5 and 6.

THEOREM 10. If $A X B$ is an arc and $J$ is a simple closed curve separating $A$ from $B$, then $J+A X B$ contains a simple closed curve $J_{1}$ separating $A$ from $B$ such that $J_{1} \cdot A X B$ is connected.

Proof. Let $A_{1}$ and $B_{1}$ denote the first and last points respectively that the $\operatorname{arc} A X B$ has in common with $J$. Let $A Y B$ denote an arc from $A$ to $B$ not containing any point of the interval $A_{1} B_{1}$ of $A X B$. Let $G$ denote the collection of all simple closed curves $C$ in $J+A X B$ such that $C \cdot A X B$ is connected and $C \cdot A Y B$ is not vacuous. The collection $G$ is finite. Let $K$ denote $A_{1} B_{1}+\left(J-J \cdot G^{*}\right) . \dagger$ The point set $K$ is a continuum containing no point of $A Y B$; hence, $K$ does not separate $A$ from $B$. Since the common part of any two elements of $G$ is a subset of $K$ and the common part of $K$ with an element of $G$ is connected, if no simple closed curve of the collection $G$ separated $A$ from $B$, then by Theorem $9, K+G^{*}$ would not separate $A$ from $B$. But $K+G^{*}$ contains $J$ and separates $A$ from $B$. Therefore some element $J_{1}$ of $G$ separates $A$ from $B$.

Definitions. Suppose that $P$ is a point of a domain $Q$, and $\Delta$ is a collection of continua whose sum separates $P$ from the boundary of $Q$ such that each continuum of $\Delta$ lies in a component of $Q-P$ whose boundary contains $P$ but no component of $Q-P$ contains more than one element of $\Delta$. Then (1) $\Delta$ is said to be minimal $\ddagger$ with respect to $Q$ and $P$, (2) $\Delta$ is said to minimally separate $P$ from the boundary of $Q$, and (3) if $D$ is a domain containing $P$ whose boundary is a subset of $\Delta^{*}, \Delta$ is said to surround $D$ minimally with respect to $Q$ and $P$.

THEOREM 11. If $\Delta$ is a collection of continua which surrounds a connected domain $D$ minimally with respect to a domain $Q$ and $a$ point $P$, then (a) each component of $Q-P$ whose boundary contains $P$, contains one and only one element of $\Delta$, and (b) no component of $D-P$ has boundary points in more than one element of $\Delta$.

Proof. Consider (a). By definition, if $C$ is a component of $Q-P$ whose boundary contains $P$, then $C$ contains not more than one element of $\Delta$. But since $S-P$ is connected, the boundary of $C$ also contains a point of the boundary of $Q$; hence $C$ must contain at least one element of $\Delta$. Therefore $C$ contains one and only one element of $\Delta$.

\footnotetext{
† If $G$ denotes a collection of point sets, $G^{*}$ will be used to denote the sum of the elements of $G$.

$\ddagger$ The term minimal is used here because if $\Delta$ were finite no collection of fewer elements would separate $P$ from the boundary of $Q$.
} 
Next consider (b). Suppose that there exists a component $I$ of $D-P$ which has boundary points in more than one element of $\Delta$. Let $C$ denote the component of $Q-P$ which contains $I$. Then $C$ contains more than one element of $\Delta$. But since $D$ is connected, the boundary of $C$ contains $P$. This is a contradiction of (a).

THEOREM 12. If $P$ is a point of a domain $Q$, not $S$, there exist in $Q$ a finite collection $\Delta$ of continua and a connected domain $D$ containing $P$ such that $\Delta$ surrounds $D$ minimally with respect to $Q$ and $P$.

Proof. Let $D_{1}$ denote a domain lying in $Q$ and containing $P$ whose boundary is a subset of the sum of the elements of a finite collection $\Delta_{1}$ of continua lying in $Q-D_{1}$. Let $N$ denote the sum of all the continua of $\Delta_{1}$ which lie in components of $Q-P$ whose boundaries contain $P$. If $H$ and $K$ are components of $N$ lying in the same component $C$ of $Q-P$, let $T$ denote an arc in $C$ from a point of $H$ to a point of $K$. Let $\Delta$ denote the collection of all components of the point set obtained by adding $N$ to the sum of the $\operatorname{arcs} T$. The collection $\Delta$ minimally separates $P$ from the boundary of $Q$. Let $D$ denote the component of $Q-\Delta^{*}$ which contains $P$. Then the boundary of $D$ is a subset of $\Delta^{*}$ and hence $\Delta$ surrounds $D$ minimally with respect to $Q$ and $P$.

Definition. If a domain is connected and contains one of the complementary domains of each simple closed curve lying in it, then it is said to be simply connected.

Definition. If $D$ is a connected domain and $D$ contains one of the complementary domains of each simple closed curve lying in $\bar{D}$, then $\bar{D}$ is said to be simply connected and $D$ is said to be strongly simply connected.

THEOREM 13. Space is simply connected.

THEOREM 14. If $D$ is a connected domain and there exist a collection $\Delta$ of continua, a domain $Q$, and a point $P$ of $D$, such that $\Delta$ surrounds $D$ minimally with respect to $Q$ and $P$, then $D$ is simply connected.

Proof. Suppose, on the contrary, that there exists in $D$ a simple closed curve $J$ such that neither of its complementary domains, $I$ and $E$, is a subset of $D$. Since $J$ lies in $D$, both $I$ and $E$ contain points of $D$ and, therefore, points of the boundary $\beta$ of $D$. Let $T$ denote an arc lying in $S-P$ irreducible from $\beta \cdot I$ to $\beta \cdot E$. The segment $T$ intersects $J$ and, hence, is a subset of $D$. Therefore $T$ lies in $Q-P$ and contains points of two different continua of $\Delta$. This contradicts Theorem 11.

THEOREM 15. If $P$ is a point of a domain $Q$, there exists in $Q$ a simply connected domain $D$ containing $P$. 
Theorem 15 is a consequence of Theorems 12,13 , and 14 .

THEOREM 16. If $D$ is a connected domain, every component of $S-\bar{D}$ is strongly simply connected.

Proof. Let $C$ denote a component of $S-\bar{D}$. Let $J$ denote a simple closed curve lying in $\bar{C}$, and let $I$ denote the interior of $J$ with respect to a point $\omega$ of $D$. Suppose that $X$ is a point of $\bar{D}$ and belongs to $I$. Then $X+I$ is a connected point set containing $\omega$ and a point of $I$ but no point of $J$. This is a contradiction.

THEOREM 17. If $D$ is a complementary domain of a locally compact continuous curve $M$ and $P$ is a point of the boundary of $D$, then $K=D+P$ is a connected, connected im kleinen inner limiting set.

Proof. It is evident that $K$ is a connected inner limiting set. It is furthermore evident that $K$ is connected im kleinen at every one of its points with the possible exception of $P$. Suppose that $K$ is not connected im kleinen at P. Then there exists a simply connected domain $Q$ containing $P$ such that (1) the component $C$ of $Q \cdot K$ that contains $P$ is not open with respect to $K$ at the point $P$, and (2) $Q \cdot M$ is compact. Hence there exists a sequence $\alpha$ of points of $K$ converging to $P$ such that each point of $\alpha$ belongs to some component of $Q \cdot K$ but not to $C$ and no two points of $\alpha$ belong to the same component of $Q \cdot K$. There exists a point $O$ of $K$ not belonging to $Q$, for otherwise $Q \cdot K=K$ would be connected and open with respect to $K$ at $P$. For each point $X$ of $\alpha$ let $X O$ denote an arc from $X$ to $O$ lying in $D$, and let $Y$ denote the first point in the order from $X$ to $O$ that $X O$ has in common with the boundary of $Q$.

Now let $Q_{1}$ denote a domain lying in $Q$ and containing $P$ such that $\beta_{1}$, the boundary of $Q_{1}$, is a subset of the sum of a finite collection $\Delta$ of continua lying in $Q-Q_{1}$. For infinitely many points $X$ of the sequence $\alpha$ the interval $X Y$ of the arc $X O$ contains a point of some one of the continua of the finite collection $\Delta$. Hence there exists a continuum $H$ of $\Delta$ such that for infinitely many points $X$ of $\alpha$ the $\operatorname{arc} X Y$ contains a point of $H$. Now let $D_{H}$ and $D_{P}$ denote connected domains lying in $Q$ and containing $H$ and $P$ respectively such that $\bar{D}_{H} \cdot \bar{D}_{P}=0$.

For each point $X$ of $\alpha$ such that $X$ lies in $D_{P}$ and the arc $X Y$ contains some point of $H$, let $T X$ denote an arc lying in $D_{P}$ such that $T$ belongs to $M$ but the segment $T X$ is a subset of $D$, and let $Z$ denote a point of $X Y \cdot H$. Now since $D_{H}$ lies in $Q$ and contains points of more that one component of $Q \cdot K, D_{H}$ contains some point of $M$. For each $Z$ let $Z W$ denote an arc lying in $D_{H}$ such that the segment $Z W$ is a subset of $Q$ and $W$ is a point of $M$. Thus for some infinite subsequence $x_{1}, x_{2}, x_{3}, \cdots$ of $\alpha$ there correspond sequences 
of points $T_{1}, T_{2}, T_{3}, \cdots$ and $W_{1}, W_{2}, W_{3}, \cdots$ such that (1) the points $T_{1}, T_{2}, T_{3}, \cdots$ belong to $D_{P} \cdot M,(2)$ the points $W_{1}, W_{2}, W_{3}, \cdots$ belong to $D_{H} \cdot M$, (3) for each $n, T_{n} X_{n}+X_{n} Z_{n}+X_{n} W_{n}$ contains an arc $T_{n} W_{n}$ lying in $Q$, and (4) if $m \neq n, \widehat{T_{m} W} m$ and $\widehat{T}_{n} W_{n}$ are mutually exclusive and lie in different components of $Q \cdot K$. Since $M$ is a continuous curve, $Q \cdot M$ is compact and $\bar{D}_{H} \cdot \bar{D}_{P}=0$, there exist two mutually exclusive connected subsets $d_{P}$ and $d_{H}$ of $Q \cdot M$ which are open with respect to $M$ such that for infinitely many values of $n$ and in particular for the three different values $a, b$, and $c$ of $n, T_{n}$ and $W_{n}$ lie in $d_{P}$ and $d_{H}$ respectively. Let $T_{a} T_{b}$ and $T_{a} T_{c}$ denote arcs lying in $d_{P}$ and $W_{a} W_{b}$ and $W_{a} W_{c}$ denote arcs lying in $d_{H}$. There exist three $\operatorname{arcs} A O_{a} B, A O_{b} B$, and $A O_{c} B$ lying in $T_{a} T_{b}+T_{a} W_{a}+W_{a} W_{b}, \quad T_{a} T_{b}+T_{b} W_{b}+W_{a} W_{b}$, and $T_{a} T_{c}+T_{c} W_{c}+W_{a} W_{c}$ respectively and having only their end points $A$ and $B$ in common. By Theorem 5 of Chapter III of Foundations, the sum of one pair of these three arcs, say $A O_{a} B$ and $A O_{c} B$, forms a simple closed curve $J$ lying in $Q$ and whose interior $I$ with respect to a point $\omega$ of the boundary of $Q$ contains the other segment, $\widehat{A O b B}$. Hence $I$ contains the component $C$ of $Q \cdot K$ which contains $\widehat{T}_{b} W_{b}$, for $C$ contains no point of $J$. However, $C$ has limit points in the boundary of $Q$. But since $Q$ is simply connected, $\bar{I}$ is a subset of $Q$. This is a contradiction, and $K$ is connected im kleinen at $P$.

THEOREM 18. If $D$ is a complementary domain of a locally compact continuous curve, and $P$ is a point of the boundary of $D$, then $P$ is accessible from $D$.

Theorem 18 follows immediately from Theorems 1 and 10 of Foundations and Theorem 17.

A number of the intuitive propositions of the plane concerning abutting and crossing arcs hold true. Although some of these will be used in arguments to follow, they will not be stated and the reader is referred to Chapter IV, Theorems 28-32, of Foundations for their precise statement and proof. Some of these proofs must be modified, however, to be valid for the set of axioms used here.

THEOREM 19. An arc is accessible from both sides at any interior point.

THEOREM 20. A ray separates space into at most two connected domains.

Proof. Suppose, on the contrary, that there exists a ray $\overrightarrow{P B}$ such that $S-\overrightarrow{P B}$ contains three components. There exist three $\operatorname{arcs} A_{1} B_{1}, A_{2} B_{2}$, and $A_{3} B_{3}$ no two of which contain points of the same component of $S-\overrightarrow{P B}$ such that (1) for each $i,(i=1,2,3), A_{i} B_{i} \cdot \overrightarrow{P B}=B_{i}$, and (2) $B_{1}$ belongs to the interval $P B_{2}$, and $B_{2}$ to the interval $P B_{3}$, of $\overrightarrow{P B}$. By Theorems 7 and 9 , $M=A_{1} B_{1}+A_{2} B_{2}+A_{3} B_{3}+P B_{3}$ does not separate space. By Theorem 18 the 
points $A_{1}, A_{2}$, and $A_{3}$ are accessible from $S-M$. Thus for each $i, i=1,2,3$, there exists an $\operatorname{arc} A_{i} B_{i}^{\prime}$ lying in $(S-M)+A_{i}$ and having only the point $B_{i}^{\prime}$ in common with $\overrightarrow{P B}$. For each $i, i=1,2,3$, let $J_{i}$ denote the simple closed curve composed of $A_{i} B_{i}, A_{i} B_{i}^{\prime}$ and the interval $B_{i} B_{i}^{\prime}$ of $\overrightarrow{P B}$, and let $I_{i}$ denote that complementary domain of $J_{i}$ whose common part (if any) with $\overrightarrow{P B}$ is compact. Either (1) $I_{1}$ contains $A_{2}$, or (2) $I_{3}$ contains $A_{2}$, or (3) $I_{2}$ contains $A_{1}$, or (4) $I_{2}$ contains $A_{3}$. Any one of these four possibilities leads to a contradiction. For instance, suppose that $I_{1}$ contains $A_{2}$. Since $I_{1} \cdot \overrightarrow{P B}$ is a subset of $P B_{1}$, it is clear, by Theorems 9 and 18, that $I_{1}+A_{1}$ contains an arc from $A_{1}$ to $A_{2}$ not intersecting $\overrightarrow{P B}$.

Theorem 21. If the arc $A B$ is a subset of the connected domain $D$ and $D-A B$ is connected, then $D$ contains a simple closed curve separating $A$ from $B$.

Proof. Since the $\operatorname{arc} A B$ is accessible from both sides at an interior point $O$, there exist two arcs $E O$ and $F O$ abutting on $A B$ from different sides such that neither $E O$ nor $F O$ has any point except $O$ in common with the $\operatorname{arc} A B$. The arcs $E O$ and $F O$ contain points $E_{1}$ and $F_{1}$, respectively, such that the intervals $E_{1} O$ and $F_{1} O$ of $E O$ and $F O$ respectively lie in $D$. In $D-A B$ there exists an arc $E_{1} F_{1}$, and in $E_{1} O+E_{1} F_{1}+F_{1} O$ there exists a simple closed curve $J$ which lies in $D$ and separates $A$ from $B$.

THEOREM 22. If $P$ is a point of a connected domain $Q$, then there exists a region $R$ containing $P$ such that if $X$ is a point of $R-P, X$ lies in a simple domain which together with its boundary is a subset of $Q$.

Proof. If $Q$ is $S$, the theorem is evident from Theorem 2. Suppose that the boundary of $Q$ is not vacuous and that the theorem is false.

Then there exists a sequence $\alpha$ of points $X$ of $Q-P$ converging to $P$ such that $X$ does not lie in a simple domain which together with its boundary is a subset of $Q$. Let $\rho$ denote a monotonic sequence of simply connected domains $D$ closing down on $P$. Let $D_{1}$ denote an element of $\rho$ lying in $Q$. It is clear from Axioms 3 and $5_{1}^{*}$ that $Q_{1}-P$ contains only a finite number of components. One of these components $C_{1}$ contains two points $X_{1}$ and $X_{2}$ of $\alpha$. Let $T_{1}$ denote an arc from $X_{1}$ to $X_{2}$ in $C_{1}$. Let $D_{2}$ denote the first element of $\rho$ which follows $D_{1}$ and contains no point of $T_{1}$. One of the components of $D_{2}-P$ contains two points $X_{3}$ and $X_{4}$ of $\alpha$ and an arc $T_{2}$ from $X_{3}$ to $X_{4}$. Let $D_{3}$ denote the first element of $\rho$ not containing a point of $T_{1}+T_{2}$. Then $D_{3}-P$ contains an $\operatorname{arc} T_{3}$ whose end points are points of $\alpha$. This process may be continued. Thus there exists a sequence of mutually exclusive $\operatorname{arcs} T_{1}, T_{2}, T_{3}, \cdots$ converging to $P$ such that for each $n, T_{n}$ is a subset of $D_{n}-P$ and the end points of $T_{n}$ are points of $\alpha$. By the preceding theorem, for each $n, T_{n}$ separates $D_{1}$. 
For each $n$, one of the components of $D_{1}-T_{n}$ does not contain $P$ and in this component there exists a segment $W_{n}$ having one end point in $T_{n}$ and containing no point of $T_{1}+T_{2}+\cdots+T_{n-1}$. By Theorems 7 and $9, P+T_{1}+T_{2}$ $+\cdots+T_{n}$ does not separate space. Hence there exists a segment $W_{n}^{\prime}$ lying in $D_{1}-\left(P+T_{1}+T_{2}+\cdots+T_{n}\right)$ having one end point in $W_{n}$ and the other end point in the boundary of $D_{1}$. For each $n$ let $L_{n}$ denote the component of $D_{1}-\left(P+T_{1}+T_{2}+\cdots+T_{n}\right)$ which contains $W_{n}+W_{n}^{\prime}$. Then for each $n, T_{n}$ separates $L_{n}$ from $P$ in $D_{1}$, and $\bar{L}_{n}$ contains points of both $T_{n}$ and the boundary of $D_{1}$. By Axiom $5_{1}^{*}$ there exists in $D_{1}$ a domain $U$ which contains $P$ and whose boundary is a subset of the sum of a finite number of continua lying in $D_{1}-U$. There is a continuum $H$ of this set which, for infinitely many values $j$ of $n$, contains a point of $L_{j}$ while $U$ contains $T_{j}$. But if $k$ is the smallest value of $j, P+H+\sum L_{j}$ is a connected point set in $D_{1}-T_{k}$ containing $P$ and $L_{k}$. This is a contradiction.

Definitions. If $P$ is a point and there exists a sequence of simple domains closing down on $P$, then $P$ is said to be a simple point. A non-simple point is said to be an edge point.
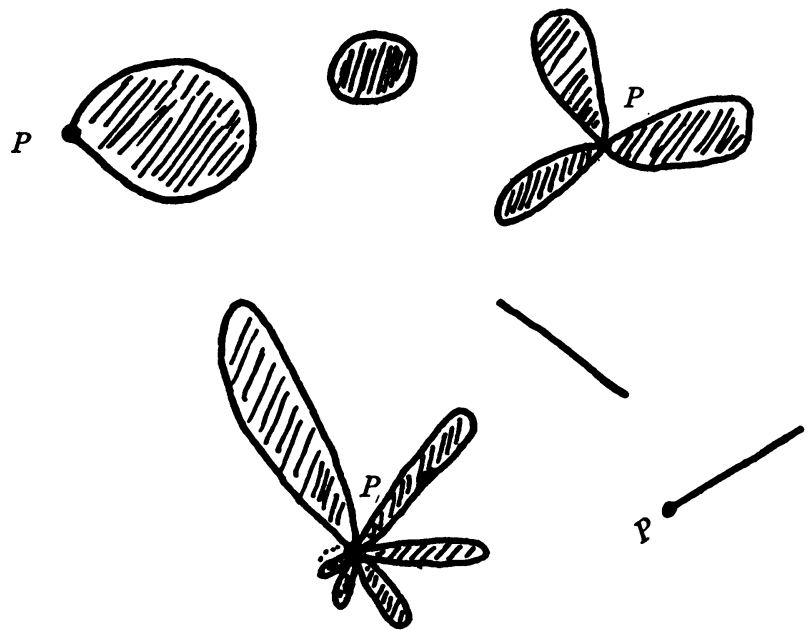

FIGURE 2

If from the plane of Figure 2 the shaded domains and their boundaries except for the points $P$ are removed, the result is a space which satisfies Axioms $0-4$ and $5_{1}^{*}$. The points $P$ are edge points while all other points are simple points.

THEOREM 23. The set of simple points is everywhere dense.

Proof. Suppose that $R$ is a region. With the help of repeated applications 
of Theorem 22 it may be shown that there exists a sequence $D_{1}, D_{2}, D_{3}, \cdots$ of simple domains lying in $R$ such that, for each $n$, (1) $D_{n}$ contains $\bar{D}_{n+1}$, and (2) $\bar{D}_{n}$ lies in some region of the collection $G_{n}$ of Axiom 1. By (4) of Axiom 1, there is a point $P$ common to $\bar{D}_{1}, \bar{D}_{2}, \bar{D}_{3}, \cdots$ and hence, to $D_{1}, D_{2}, D_{3}, \cdots$; and by (3) of Axiom 1, $P$ is the only common point. The point $P$ is simple and lies in $R$.

THEOREM 24. No completely separable point set contains uncountably many edge points.

Proof. Let $H$ denote the collection of all edge points belonging to a completely separable point set $M$. If $X$ is a point of $H$, there exists no sequence of simple domains closing down on $X$. Hence for each point $X$ of $H$, there exists an integer $n_{x}$ such that no region of $G_{n_{x}}$ of Axiom 1 contains a simple domain containing $X$. If $H$ is uncountable, there exist an integer $k$ and an uncountable subset $K$ of $H$, such that if $X$ is a point of $K, n_{x}=k$. Since $M$ is completely separable, $K$ has a point $P$ of condensation. Let $Q$ denote a connected domain containing $P$ and lying in a region of $G_{k}$ of Axiom 1. By Theorem 22 there exists in $Q$ a region $R$ containing $P$ such that every point $X$ of $R-P$ is contained in a simple domain lying in $Q$. This is clearly a contradiction.

THEOREM 25. If $M$ is a countable set of simple points, $S-M$ is arc-wise connected.

This theorem may be proved without using Axioms 4 and $5_{1}^{*}$ by a modification of R. L. Moore's proof $\dagger$ of Theorem 1 of Chapter II of Foundations. $\ddagger$ The kernel of this proof is the construction of a sequence $C_{1}, C_{2}, C_{3}, \cdots$ of simple chains of connected domains from a point $A$ to a point $B$ such that for each $n$, $C_{n}{ }^{*}$ contains $\bar{C}_{n+1}^{*}$ and the common part of the point sets $C_{1}^{*}, C_{2}^{*}, C_{3}^{*}, \cdots$ is an arc from $A$ to $B$. Now for each $n, C_{n}^{*}$ is a connected domain, and it is easy to see that no finite number of simple points disconnects a connected domain. Hence if (1) $A$ and $B$ are two distinct points of $S-M$, (2) $M=P_{1}+P_{2}$ $+\cdots+P_{n}+\cdots$, and (3) for each $n$ the chain $C_{n}$ is constructed so that $C_{n}{ }^{*}$ contains no point of $P_{1}+P_{2}+\cdots+P_{n}$, then the arc from $A$ to $B$ of Moore's construction will not contain any point of $M$.

TheOREM 26. If $M$ is a countable set of simple points, $S-M$ is cyclicly connected.

Proof. If $A$ and $B$ are any two points of $S-M$, then by Theorem 25 there exists an arc $A X B$ in $S-M$. With the help of Theorems 5, 7 and 17, and

$\dagger$ Cf. J. R. Kline, Concerning the complement of a countable infinity of point sets of a certain type, Bulletin of the American Mathematical Society, vol. 23 (1917), pp. 290-292.

$\ddagger$ See footnote p. 54 . 
Theorem 9 of Chapter II of Foundations, one may show that $S-\widehat{A X B}$ is a space satisfying Axioms $0,1,2$, and 3. Hence by Theorem 25 there exists an arc $A Y B$ lying in $S-(M+\widehat{A X B})$. The sum of the two arcs, $A X B$ and $A Y B$, is a simple closed curve lying in $S-M$ and containing $A+B$.

TheOREM 27. No compact set of edge points separates two simple points from each other.

Proof. Suppose that $A$ and $B$ are two simple points and that $M$ is a compact set of edge points. Suppose that $n$ is any fixed integer. Then there exists from $A$ to $B$ a simple chain of simple domains such that each element of the chain is a subset of some region of $G_{n}$ of Axiom 1. For suppose the contrary. Let $H_{A}$ denote the set of all points $X$ such that there exists from $A$ to $X$ a chain of simple domains whose elements are each a subset of some region of $G_{n}$. Since $A$ is a simple point, $H_{A}$ is a connected domain. This domain does not contain $B$, and its boundary $\beta$ is a subset of $M$. Hence $\beta$ is a closed and compact point set which separates $A$ from $B$. By Theorem 8 and Axiom 3, $\beta$ contains a non-degenerate continuum separating $A$ from $B$. Hence $M$ is uncountable, which contradicts Theorem 24 .

Now with the help of Theorem 9 of Chapter II of Foundations and Theorem 5 one may show that a simple domain is a space satisfying Axioms 0-4 and $5_{1}^{*}$, and that the complementary domain of a point is also such a space. Let $M=P_{1}+P_{2}+P_{3}+\cdots$. Let $C_{1}$ denote a simple chain of simple domains from $A$ to $B$ such that (1) each element of $C_{1}$ is a subset of some region of $G_{1}$, and (2) $C_{1}^{*}$ does not contain $P_{1}$. Now the boundary of the domain $C_{1}{ }^{*}$ is a closed and compact point set not separating $A$ from $B$; so there exists a simple closed curve separating it from $A+B$ (Theorem 3 in modification). Hence there exists in $C_{1}^{*}$ a simple domain $D_{1}$ containing $A+B$. Let $C_{2}$ denote a simple chain of simple domains from $A$ to $B$ such that if $c$ is an element of $D_{2}$, (1) $\bar{c}$ is a subset of some element of $C_{1},(2) c$ is a subset of some region of $G_{2}$, and (3) $c$ does not contain $P_{2}$. This process may be continued, and by Theorem 80 of Chapter I of Foundations, $C_{1}{ }^{*} \cdot C_{2}{ }^{*} \cdot C_{3}{ }^{*} \ldots$ is a continuum. Hence $M$ does not separate $A$ from $B$.

As a matter of fact the above process may be carried out along the lines of the proof of Theorem 1 of Chapter II of Foundations, so as to show that $S-M$ is arc-wise, and even cyclicly, connected.

Nevertheless, a countable but non-compact set of edge points may separate two simple points from each other as is shown by the following example. Let space consist of all points of the number plane whose ordinates are not zero together with those whose ordinates are zero but whose abscissas are rational. If $(X, Y)$ is a point whose ordinate $Y$ is not zero, then for each integer 
such that $1 / n<|Y|$, let $V_{n}(X, Y)$ denote the point set consisting of all points which lie at a distance less than $1 / n$ from $(X, Y)$. If $(X, Y)$ is a point whose ordinate $Y$ is zero and whose abscissa $X$ is rational then for each integer $n$, let $V_{n}(X, Y)$ denote the point set consisting of $(X, Y)$ together with all points which are interior to the isosceles triangles bounded by the lines whose equations are $y=1 / n, y=-1 / n, y=n(x-X)$, and $y=-n(x-X)$. These point sets $V_{n}$ shall be called regions, and for each integer $n, G_{n}$ of Axiom 1 shall denote the collection of all of the point sets $V_{n+q}, q=0,1,2, \cdots$. It can be shown that Axioms $0-4$ and $5_{1}^{*}$ hold true in this space. Furthermore, the space is completely separable and locally compact at all but a countable nowheredense set of points. Let $M$ denote the set of all points whose abscissas are rational and whose ordinates are zero. Each point of $M$ is an edge point. If $A$ and $B$ are points with positive and negative ordinates respectively, $M$ separates $A$ from $B$, and the point set $H_{A}$ of the above argument consists of all points whose ordinates are positive.

THEOREM 28. No compact set of edge points separates space.

Theorem 28 is a consequence of Theorems 23 and 27.

Definitions. If $D_{1}$ and $D_{2}$ are domains such that $D_{1}$ contains $\bar{D}_{2}$, and $\widehat{T}$ is a segment lying in $D_{1}-\bar{D}_{2}$ having one end point on the boundary of $D_{1}$ and the other on the boundary of $D_{2}$, then $\widehat{T}$ is said to cross $D_{1}-\bar{D}_{2}$. Furthermore, if $C$ is the component of $D_{1}-\bar{D}_{2}$ which contains $\widehat{T}$, then $\widehat{T}$ is said to cross $C$. Also the $\operatorname{arc} T$ is said to cross $D_{1}-\bar{D}_{2}$ and to cross $C$.

Definition. If $D_{1}$ and $D_{2}$ are domains such that $D_{1}$ contains $\bar{D}_{2}, C$ is a component of $D_{1}-\bar{D}_{2}$, and $\widehat{T}_{1}, \widehat{T}_{2}$, and $\widehat{T}_{3}$ are segments crossing $C$, then if $\widehat{T}_{2}$ separates $\widehat{T}_{1}$ from $\widehat{T}_{3}$ in $C, \widehat{T}_{2}$ is said to be between $\widehat{T}_{1}$ and $\widehat{T}_{3}$ in $C$, and $T_{2}$ is said to be between $T_{1}$ and $T_{3}$ in $C$.

Theorem 29. Suppose that $D_{1}$ and $D_{2}$ are simply connected domains such that $D_{1}$ contains $\bar{D}_{2}$ but no simple domain lying in $D_{1}$ contains $D_{2}, C$ is a component of $D_{1}-\bar{D}_{2}$, and $\widehat{T}_{1}, \widehat{T}_{2}$, and $\widehat{T}_{3}$ are three mutually exclusive segments crossing $C$. Then one and only one of these segments is between the other two in $C$.

Proof. That not more than one of these segments lies between the other two in $C$ is an immediate consequence of a well known theorem.

Suppose that no one of them is between the other two in $C$. Then either (1) $C-\left(\widehat{T}_{1}+\widehat{T}_{2}+\widehat{T}_{3}\right)$ contains a component $W$ which has limit points in each of the three segments, or (2) $C-\left(\widehat{T}_{1}+\widehat{T}_{2}+\widehat{T}_{3}\right)$ contains three mutually exclusive components $W_{12}, W_{13}$, and $W_{23}$ having limit points in the segments indicated by their subscripts. 
Case I. Suppose (1) holds. Then there exists in $W$ three mutually exclusive segments $\overparen{A A}_{1}, \widehat{A A}_{2}$, and $\overparen{A A}_{3}$ having a common end point $A$ in $W$ such that $A_{1}, A_{2}$, and $A_{3}$ belong to $\widehat{T}_{1}, \widehat{T}_{2}$, and $\widehat{T}_{3}$ respectively. For each $i$, $i=1,2,3$, let $O_{i}$ and $P_{i}$ denote the end points of $T_{i}$ which belong to the boundaries of $D_{1}$ and $D_{2}$ respectively. Since $D_{2}$ is connected, there exist in $D_{1}-\left(\widehat{T}_{1}+\widehat{T}_{2}+\widehat{T}_{3}+A A_{1}+A A_{2}+A A_{3}\right)$ three mutually exclusive segments $\widehat{B B}_{1}, \widehat{B B}_{2}$, and $\overparen{B B}_{3}$ having a common end point $B$ in $D_{2}$ and having their other end points $B_{1}, B_{2}$, and $B_{3}$ in the intervals $A_{1} P_{1}$ of $T_{1}, A_{2} P_{2}$ of $T_{2}$, and $A_{3} P_{3}$ of $T_{3}$ respectively. If $A B_{n} B$ denotes $A A_{n}+A_{n} B$ (of $\left.A_{n} P_{n}\right)+B B_{n}$, $n=1,2,3$, then the segments $A B_{1} B, A B_{2} B$, and $A B_{3} B$ are mutually exclusive. By Theorem 5 of Chapter III of Foundations, the sum of two of these arcs, say $A B_{1} B$ and $A B_{3} B$, forms a simple closed curve $J$ whose interior $I$ with respect to $O_{1}$ contains the segment from $A$ to $B$ of the other one. Since $D_{1}$ is simply connected, $I$ is a subset of $D_{1}$. But $T_{2}$ contains $B_{2}$ and no point of $J$ and is, therefore, a subset of $I$. Hence $I$ contains $O_{2}$. This is.a contradiction.

Case II. Suppose (2) holds. There exist segments $\widehat{A_{1} B_{3}}, \widehat{B_{2} A_{3}}$, and $\widehat{B_{1} A_{2}}$ lying in $W_{13}, W_{23}$, and $W_{12}$ respectively and having end points on the arcs $T_{1}, T_{2}$, and $T_{3}$ as indicated by the subscripts in the notation. The point set $A_{1} B_{3}+A_{1} B_{1}$ (of $\left.T_{1}\right)+B_{1} A_{2}$ (of $\left.T_{2}\right)+B_{2} A_{3}+A_{3} B_{3}$ (of $T_{3}$ ) is a simple closed curve $J$ lying in $D_{1}-D_{2}$. Since $D_{1}$ is simply connected, one of the complementary domains $I$ of $J$ is a subset of $D_{1}$. The domain $I$ either contains $D_{2}$ or is a subset of $D_{1}-\bar{D}_{2}$. By hypothesis $D_{1}$ contains no simple domain containing $D_{2}$. Hence $I$ is a subset of $C-\left(\widehat{T}_{1}+\widehat{T}_{2}+\widehat{T}_{3}\right)$ and has limit points in each of the three segments $\widehat{T}_{1}, \widehat{T}_{2}$, and $\widehat{T}_{3}$, which is Case I again.

The reader can see that any collection of mutually exclusive segments crossing $C$ would, in accordance with this notion of between, have a linear order in $C$.

Theorem 30. Suppose that $D_{1}, D_{2}$, and $D_{3}$ are simply connected domains containing the point $P$ such that (1) $D_{3}$ contains $\bar{D}_{2},(2) D_{3}$ is surrounded minimally with respect to $D_{1}$ and $P$ by a finite collection $\Delta$ of continua lying in $D_{1}-D_{3}$, and (3) $D_{1}$ contains no simple domain containing $P$. Then if $C$ is a component of $D_{1}-\bar{D}_{2}, T$ is an arc crossing $C$, and $J$ is a simple closed curve separating $T$ from $P, J$ contains two arcs $T_{a}$ and $T_{b}$ which cross $C$ such that $T$ is between them in $C$.

Proof. Let $R$ denote a connected domain lying in $D_{1}-D_{1} \cdot J$ and containing the end point $A$ of $T$ which is on the boundary of $D_{2}$. Now in $R+D_{2}$ there exists an $\operatorname{arc} A P$ from $A$ to $P$, such that $J \cdot A P$ is a subset of an interval $A^{\prime} P^{\prime}$ of $A P$ lying in $D_{2}$. By Theorem $10, J+A P$ contains a simple closed curve $J_{1}$ 
separating $A$ from $P$ such that $J_{1} \cdot A P$ is connected; but $J_{1} \cdot A P$ is a subset of $A^{\prime} P^{\prime}$ and is, therefore, a subset of $D_{2}$. Since $D_{1}$ is simply connected but contains no simple domain containing $P, J_{1}$ contains a point $O$ not belonging to $D_{1}$. The point set $J_{1}-\left(J_{1} \cdot A P+O\right)$ is the sum of two mutually exclusive segments $\overparen{O X}$ and $\overparen{O Y}$, where $X$ and $Y$ belong to $A P$ (and $Y$ belongs to the interval $X P$ of $A P$ ) and the arcs $O X$ and $O Y$ abut on $A P$ from different sides. Both $O X$ and $O Y$ contain arcs which cross $D_{1}-\bar{D}_{2}$. Let $T_{a}$ denote the first interval of $O X$ in the order from $X$ to $O$ that crosses $D_{1}-\bar{D}_{2}$, and let $T_{b}$ denote the first interval of $O Y$ in the order from $Y$ to $O$ which crosses $D_{1}-\bar{D}_{2}$. Now $C$ contains an element of $\Delta$ and if $U$ denotes the component of $D_{1}-P$ which contains $C, U$ has no point in common with any other component of $D_{1}-\bar{D}_{2}$ because $\Delta$ is a minimal collection with respect to $D_{1}$ and $P$. Hence $T_{a}$ and $T_{b}$ cross $C$. Let $X^{\prime}$ and $Y^{\prime}$ denote the end points of $T_{a}$ and $T_{b}$ respectively which are on the boundary of $D_{2}$. The intervals $X X^{\prime}$ of $O X$ and $Y Y^{\prime}$ of $O Y$ lie in $D_{1}$.

Suppose that $T$ is not between $T_{a}$ and $T_{b}$ in $C$. Then there exists an $\operatorname{arc} T_{1}$ in $C$ irreducible from $T_{a}$ to $T_{b}$ not intersecting $T$. Let $W$ denote the last point that $O X$ has in common with $T_{1}$, and let $Z$ denote the last point that $O Y$ has in common with $T_{1}$. The intervals $W X$ of $O X$ and $Z Y$ of $O Y$ are subsets of $T_{a}+X X^{\prime}$ and $T_{b}+Y Y^{\prime}$ respectively, and hence, are subsets of $D_{1}$; furthermore, $W X$ and $Z Y$ abut on $A P$ from different sides. Now $J_{2}=J_{1} \cdot A P+W X$ $+W Z$ (of $\left.T_{1}\right)+Z Y$ is a simple closed curve lying in $D_{1}$ not containing $P$, or a point of $T$, or a point of the segment $\overparen{P Y}$ of $A P$. Since $D_{1}$ is simply connected, one of the complementary domains $I$ of $J_{2}$ is a subset of $D_{1}$. But since $D_{1}$ does not contain a simple domain containing $P$, both $P$ and $T$ must be subsets of the other complementary domain $E$ of $J_{2}$. Now $A X$ contains an arc which abuts on $J_{2}$ from the side opposite $P Y$ of $A P$, and since both $A$ and $\overparen{P Y}$ are in $D, \overparen{A X}$ must intersect $J_{2}$. Let $A_{1}$ and $A_{2}$ denote the first and last points respectively that $\overparen{A X}$ has in common with $J_{2}$. The segments $\overparen{A A_{1}}$ and $\overparen{A_{2} X}$ of $A X$ lie in $E$ and $I$ respectively. The segment $\widehat{A X}$ does not intersect $J_{1}$; so $\overparen{A X} \cdot J_{2}$ is a subset of $T_{1}$. Hence $\widetilde{A A}{ }_{1}$ and $\overparen{A_{2} X}$ abut on $T_{1}$ from different sides. Let $R^{\prime}$ denote a connected domain lying in $D_{1}$ and containing $A$ but no point of $T_{1}+T_{a}+T_{b}$. Then in $R^{\prime}+D_{2}$ there exists an arc $F G$ irreducible from $A A_{1}$ to $A_{2} X$. The simple closed curve $J_{3}=F G+G A_{2}\left(\right.$ of $\left.A_{2} X\right)+A_{1} A_{2}\left(\right.$ of $\left.T_{1}\right)+A_{1} F$ (of $\left.A A_{1}\right)$ lies in $D_{1}$, contains no point of $T_{a}+T_{b}$, but crosses the arc $T_{1}$. Hence, $T_{a}$ and $T_{b}$ lie in different complementary domains of $J_{3}$. This is impossible, for since $D_{1}$ is simply connected, one complementary domain of $J_{3}$ is a subset of $D_{1}$.

THEOREM 31. In order that space be metric, it is necessary and sufficient that space be completely separable. 
Proof. That a space satisfying Axioms 0 and 1 is metric if it is completely separable has been shown by R. L. Moore. $\dagger$ Hence, this space is metric if it is completely separable.

It will now be shown that if space is metric, it is completely separable. Let $P$ denote an edge point, and let $R$ denote a region containing $P$ but containing no simple domain containing $P$. Since $R$ is not $S$, by Theorems 12 , 14 , and 15 , there exist in $R$ three simply connected domains $D_{1}, D_{2}$, and $D_{3}$ such that $D_{3}$ contains $\bar{D}_{2}$, and $D_{3}$ is surrounded minimally with respect to $D_{1}$ and $P$ by a collection $\Delta$ of continua lying in $D_{1}-D_{3}$. The domain $D_{1}$ contains no simple domain containing $P$. Let $C$ denote a component of $D_{1}-\bar{D}_{2}$.

Suppose that there are two mutually exclusive $\operatorname{arcs} T_{1}$ and $T_{-1}$ crossing $C$. Let $\alpha$ denote a well-ordered sequence (whose first element is $T_{1}$ ) of all $\operatorname{arcs} T$ which cross $C$ such that either (1) $T$ is $T_{1}$, or (2) $T_{1}$ lies between $T_{-1}$ and $T$ in $C$. Let $\alpha^{\prime}$ denote a subsequence of $\alpha$ such that (1) the first element of $\alpha^{\prime}$ is $T_{1}$ and (2) if an element $T$ of $\alpha^{\prime}$ is not $T_{1}$, then $T$ is the first element of $\alpha$ which neither intersects a preceding element of $\alpha^{\prime}$ nor lies between two preceding elements of $\alpha^{\prime}$. Every element of $\alpha$ either intersects an element of $\alpha^{\prime}$ or lies between $T_{1}$ and some element of $\alpha^{\prime}$, and between any element of $\alpha^{\prime}$ and the next following element in $\alpha^{\prime}$ there is no element of $\alpha^{\prime}$.

The sequence $\alpha^{\prime}$ is countable. For suppose that $\alpha^{\prime}$ is uncountable. For each arc $T$ of $\alpha^{\prime}$ let $d_{T}$ denote the distance from $T$ to the next element of $\alpha^{\prime}$. There exist a number $\epsilon$ and an uncountable subsequence $\alpha_{\epsilon}^{\prime}$ of $\alpha^{\prime}$ such that if $T$ belongs to $\alpha_{\epsilon}^{\prime}, d_{T}>\epsilon$. Let $T_{2}$ denote the first element of $\alpha^{\prime}$ such that $T$ is preceded in $\alpha^{\prime}$ by infinitely many elements of $\alpha_{\epsilon}^{\prime}$. Let $L$ denote an arc from $T_{1}$ to $T_{2}$ lying in $C$. Infinitely many elements of $\alpha_{\varepsilon}^{\prime}$ are between $T_{1}$ and $T_{2}$ in $C$ and therefore intersect $L$. Consequently, there exists in $C$ a connected domain $D$ of diameter less than $\epsilon / 2$ which contains points of two different elements $T$ and $T^{\prime}$ of $\alpha_{\epsilon}^{\prime}$, such that $T$ precedes $T^{\prime}$ in $\alpha^{\prime}$. Then, the first element of $\alpha^{\prime}$ which follows $T$ in $\alpha^{\prime}$ is either $T^{\prime}$ or lies between $T$ and $T^{\prime}$ in $C$ and intersects $D$, which is a contradiction. The same may be done for the collection of all $\operatorname{arcs} T$ such that $T_{-1}$ lies between $T_{1}$ and $T$ in $C$. Hence there exists a countable collection $G$ of arcs crossing $C$, such that any arc which crosses $C$ either intersects an arc of $G$ or lies between two arcs of $G$. This was on the assumption that there exist two mutually exclusive arcs crossing $C$. It is evident that $G$ exists if this is not the case, for there exists at least one arc which crosses $C$.

For each pair of arcs of $G$ let $L$ denote an arc in $C$ which contains points of both of them, and let $M_{c}$ denote the sum of all these arcs together with the sum of the elements of $G$. Then if $T$ is any arc which crosses $C, T$ intersects

† See Foundations, p. 464. 
$M_{c}$. Since each component $C$ of $D_{1}-\bar{D}_{2}$ contains an element of $\Delta$, there are only a finite number of them. Hence $M=\overline{\sum M}_{c}$ is a closed and completely separable subset of $\bar{D}_{1}-P$ which separates $P$ from the boundary of $D_{1}$. Let $Q$ denote the component of $\bar{D}_{1}-M$ which contains $P$. Then $Q$ contains $P$ and lies in $R$, and the boundary of $Q$ is completely separable. Thus, if $P$ is an edge point of a region $R$, there exists in $R$ a domain containing $P$ whose boundary is separable. This is also true if $P$ is a simple point of a region $R$. Therefore, space is locally peripherally separable. By a theorem of the author's such a connected, connected im kleinen metric space is completely separable. $\dagger$

THEOREM 32. If space is completely separable and $P$ is a point, there exists a sequence of simple domains $Q_{1}, Q_{2}, Q_{3}, \cdots$ bounded by simple closed curves $J_{1}, J_{2}, J_{3}, \cdots$ respectively, such that (1) for each $n, Q_{n}$ contains $\bar{Q}_{n+1}$, and (2) if $M$ is a closed and compact point set not containing $P$, there exists an integer $n$ such that $\bar{Q}_{n}$ does not contain a point of $M$.

Proof. Let $G$ denote the collection of all simple domains $D$ such that $\bar{D}$ is a subset of $S-P$. By Theorem 2, $G$ covers $S-P$, and since space is completely separable, $G$ contains a countable subcollection $D_{1}, D_{2}, D_{3}, \cdots$ which covers $S-P$. For each $n$ let $C_{n}$ denote the boundary of $D_{n}$. Let $J_{1}$ denote the boundary of a simple domain containing $P$. For each $n, n \geqq 2$, let $J_{n}$ denote a simple closed curve separating $P$ from the closed and compact point set $C_{1}+C_{2}+\cdots+C_{n-1}+J_{1}+J_{2}+\cdots+J_{n-1}$, and let $Q_{n}$ denote the complementary domain of $J_{n}$ which contains $P$. Then $Q_{1}, Q_{2}, Q_{3}, \cdots$ is the required sequence of simple domains. For if $M$ is any closed and compact point set, there exists an integer $k$ such that $D_{1}+D_{2}+\cdots+D_{k}$ covers $M$. Hence, if $n>k, \bar{Q}_{n}$ contains no point of $M$.

Definition. Suppose that $D$ is a connected domain. If there exist a collection $\Delta$ of open curves, a simply connected domain $Q$, and a point $P$ of $D$, such that (1) $Q$ contains no simple domain containing $P$, and (2) $\Delta$ surrounds $D$ minimally with respect to $Q$ and $P$, then $D$ is said to be pseudo-simple, and in particular, $D$ is said to be pseudo-simple with respect to $Q$ and $P$.

TheOREM 33. If space is completely separable and $D_{1}$ is a simply connected domain containing an edge point $P$ but containing no simple domain containing $P$, then $D_{1}$ contains a domain $D$ which is pseudo-simple with respect to $D_{1}$ and $P$.

Proof. Let $D_{2}$ and $D_{3}$ denote simply connected domains containing $P$ such that (1) $D_{3}$ contains $\bar{D}_{2}$, and (2) $D_{3}$ is surrounded minimally with respect to $D_{1}$

$\dagger$ A theorem concerning locally peripherally separable spaces, Bulletin of the American Mathematical Society, vol. 41 (1935), pp. 437-439. 
and $P$ by a finite collection $\Delta^{\prime}$ of continua lying in $D_{1}-D_{3}$. By Theorem 32 there exists a sequence of simple domains $Q_{1}, Q_{2}, Q_{3}, \cdots$ bounded by simple closed curves $J_{1}, J_{2}, J_{3}, \cdots$ respectively, such that (1) for each $n, Q_{n}$ contains $\bar{Q}_{n+1}$, and (2) if $M$ is a closed and compact subset of $S-P$, there exists an integer $n$ such that $\bar{Q}_{n}$ contains no point of $M$. Let $C$ denote a component of $D_{1}-\bar{D}_{2}$, and let $G$ denote the collection of all arcs $T$ such that $T$ crosses $C$ and for some $n$ is a subset of $J_{n}$. If $T$ and $T^{\prime}$ are arcs of $G, \widehat{T} \cdot \widehat{T}^{\prime}=0$. Since $D_{1}$ is simply connected but contains no simple domain containing $P$, for each $n, J_{n}$ contains a point of $S-D_{1}$. Furthermore, let $A P$ denote an arc lying in $D_{2}$ and in the component of $D_{1}-P$ which contains $C$; then there exists an integer $n_{1}$ such that $\bar{Q}_{n_{1}}$ does not contain $A$; so if $n>n_{1}, J_{n}$ contains a point of the segment $\overparen{A P}$ and a point of $S-D_{1}$, and therefore contains at least two $\operatorname{arcs}$ of $G$. However for each $n, J_{n}$ contains at most a finite number of $\operatorname{arcs}$ of $G$. Hence $G$ is countably infinite. Furthermore, suppose that $L$ is an arc lying in $C$ from one arc of $G$ to another arc of $G$. There exists an integer $n_{2}$ such that if $n>n_{2}, \bar{Q}_{n}$ contains no point of $L$. Hence, between any two $\operatorname{arcs}$ of $G$ there are only a finite number of arcs of $G$. Each arc of $G$ is between some two arcs of $G$. For, if $T$ is an arc of $G$, there exists an integer $n$ such that $J_{n}$ separates $T$ from $P$. By Theorem 30, $J_{n}$ contains two arcs which cross $C$ such that $T$ is between them in $C$.

Let $\alpha$ denote a well-ordered sequence whose elements are the elements of $G$. Let $N_{1}$ and $N_{0}$ denote the first two elements of $\alpha$. Let $N_{2}$ denote the first element of $\alpha$ such that $N_{1}$ is between $N_{0}$ and $N_{2}$ in $C$. Let $N_{-1}$ denote the first element of $\alpha$ such that $N_{0}$ is between $N_{-1}$ and $N_{1}$ in $C$. This process may be continued. The sequences $N_{1}, N_{2}, N_{3}, \cdots, N_{\omega}, \cdots$ and $N_{0}, N_{-1}, N_{-2}, \cdots, N_{-\omega}, \cdots$ may or may not be simple sequences, but since each is countable, the first contains a simple countable subsequence $T_{1}, T_{2}, T_{3}, \cdots$ running through it and the second contains a simple countable subsequence $T_{0}, T_{-1}, T_{-2}, \cdots$ running through it, such that if $T$ is any arc crossing $C$, there exists an integer $n$ such that $T$ is between $T_{n}$ and $T_{-n}$ in $C$, and such that the linear order of these arcs in $C$ is the same as the order in the sequences.

Since $C$ contains one and only one of the continua of $\Delta^{\prime}$ and by Theorem 31 space is metric, there exists in $C$ a connected domain $C^{\prime}$ such that (1) $C^{\prime}$ contains this element of $\Delta^{\prime}$, and (2) $\bar{C}^{\prime}$ is a subset of $C$. For each pair of consecutive integers (positive or negative), $a$ and $b$ with $a<b$, let $U_{a}$ denote an arc irreducible from $T_{a}$ to $T_{b}$ lying in $C^{\prime}$, and let $M_{a}$ denote the interval of $T_{a}$ between the end points of $U_{a}$ and $U_{a-1}$ in $T_{a}$. The point set $\sum M_{a}$ is closed, since each $M_{a}$ is for some $n$ a closed subset of $C \cdot J_{n}$, for each $n J_{n}$ contains only a finite number of the arcs of $G$, and the limiting set of $J_{1}, J_{2}, J_{3}, \cdots$ is 
the point $P$. Also $\sum U_{a}$ is closed; for suppose that $O$ is a limit point of the set $\sum U_{a}$ not belonging to it. The point $O$ belongs to $\bar{C}^{\prime}$ and therefore, to $C$. There exist a connected domain $V$ lying in $C$ and containing $O$ but not intersecting more than one of the simple closed curves $J_{1}, J_{2}, J_{3}, \cdots$, and an infinite collection $H$ of segments $\widehat{U}_{a}$ such that if $\widehat{U}_{a}$ is a segment of $H, \widehat{U}_{a}$ intersects $V$. Hence, $V+H^{*}$ is a connected subset of $C$ not intersecting more than one of the $\operatorname{arcs} T_{1}, T_{2}, T_{3}, \cdots, T_{0}, T_{-1}, T_{-2}, \cdots$, but having limit points in infinitely many of them. This is a contradiction. So $L=\sum\left(M_{a}+U_{a}\right)$ is closed and is, because of its method of construction, an open curve.

Now suppose that $T$ is any arc crossing $C$. There exists an integer $n$ such that $T$ lies between $T_{n}$ and $T_{-n}$ in $C$. Hence $T$ intersects that interval of $L$ which is irreducible from $T_{n}$ to $T_{-n}$. Furthermore, if this process is carried out in each component of $D_{1}-\bar{D}_{2}$ and $\Delta$ denotes the collection of open curves. so obtained, $\Delta^{*}$ separates $P$ from the boundary of $D_{1}$, and since no component of $D_{1}-P$ contains more than one element of $\Delta^{\prime}$, no component contains more than one element of $\Delta$. Hence, $\Delta$ is minimal with respect to $D_{1}$ and $P$.

Let $D$ denote the component of $S-\Delta^{*}$ which contains $P$. The domain $D$ is pseudo-simple with respect to $D_{1}$ and $P$.

THEOREM 34. If $L$ is an element of a collection of open curves which surrounds a pseudo-simple domain $D$ minimally with respect to a simply connected. domain $Q$ and a point $P$, and $C$ is the component of $D-P$ whose boundary contains a point of $L$, then no ray of $L$ separates $C$ from the boundary of $Q$ in $S-P$.

Proof. Suppose the contrary is true. Then $L$ contains a ray $\overrightarrow{B_{1} B_{2}}$ such that $B_{1}$ belongs to $\bar{C}$ and $\overrightarrow{B_{1} B_{2}}+P$ separates $C$ from $\beta$, the boundary of $Q$. By Theorem $18, C+P+B_{1}$ contains an arc $P B_{1}$ from $P$ to $B_{1}$. The ray $\overrightarrow{P B}_{2}=P B_{1}+{\overrightarrow{B_{1} B_{2}}}_{2}$ separates $C-C \cdot P B_{1}$ from $\beta$, and by Theorem $20, S-\overrightarrow{P B}_{2}$ is the sum of two connected domains $I$ and $E$ where $I$ contains $C-C \cdot \overrightarrow{P B}_{2}$ and is a subset of $Q$ and $E$ contains $\beta$. Let $E_{1} F_{1}$ and $E_{2} F_{2}$ denote two arcs in $C$ which abut on $P B_{1}$ from different sides. The points $E_{1}$ and $E_{2}$ both belong to $C-C \cdot P B_{1}$ and, hence, to $I$. Therefore $I$ contains an arc $E_{1} E_{2}$ from $E_{1}$ to $E_{2}$, and $E_{1} E_{2}+E_{1} F_{1}+F_{1} F_{2}$ (of $\left.P B_{1}\right)+E_{2} F_{2}$ contains a simple closed curve lying in $Q$ one of whose complementary domains lies in $Q$ and contains $P$. This is a contradiction.

THEOREM 35. If $L$ is an element of a collection $\Delta$ of open curves which surrounds a pseudo-simple domain $D$ minimally with respect to a simply connected domain $Q$ and a point $P$, then no two segments lying in $D$ abut on $L$ from different sides. $\dagger$

$\dagger$ Two segments abut on a ray or open curve from different sides provided the ray or open curve contains an arc on which the segments abut from different sides. 
Proof. Suppose, on the contrary, that $D$ contains two mutually exclusive segments which abut on $L$ from different sides. It follows, using Theorem 11(b), that there exist an arc $A_{1} A_{2}$ and two segments, $\widetilde{A_{1} B_{1}}$ and $\overparen{A_{2} B_{2}}$, all lying in the same component $C$ of $D-P$, such that the segments $\widetilde{A_{1} B_{1}}$ and $\widetilde{A_{2} B_{2}}$ abut on $L$ from different sides and the point set $J=A_{1} B_{1}+B_{1} B_{2}$ (of $\left.L\right)+A_{2} B_{2}$ $+A_{1}$ is a simple closed curve lying in $Q$. One of the complementary domains of $J$ is a subset of $Q$. Hence, $L$ contains two rays, $\overrightarrow{B_{1} B_{2}}$ and $\overrightarrow{B_{2} B_{1}}$, whose common part is the arc $B_{1} B$, one of which has the property that it together with $P$ separates $C$ from the boundary of $Q$. This is a contradiction of the preceding theorem.

THEOREM 36. If $D$ is a pseudo-simple domain with respect to a simply connected domain $Q$ and a point $P, \beta$ is the boundary of $D$, and $J$ is a simple closed curve lying in $\bar{D}$ such that $J \cdot \beta$ is connected, then one of the complementary domains of $J$ is a subset of $D$.

Proof. Let $\Delta$ denote a collection of open curves which surrounds $D$ minimally with respect to $Q$ and $P$, let $L$ denote the element of $\Delta$ which contains $J \cdot \beta$, and let $C$ denote the component of $D-P$ which contains $J \cdot(D-P)$. By Theorems 7 and $9, J \cdot \beta+P$ does not separate space, and there exists an arc $T$ from a point of $L-J \cdot L$ to a point on the boundary of $Q$ which contains no point of $J$. One of the complementary domains $I$ of $J$ is a subset of $Q$. Therefore, $I$ contains no point of $T$ and, with the help of Theorem 35, it may be shown that $I$ contains no point of $L$. But $I$ does not contain $P$. $\dagger$ Hence, since $I$ contains points of $C, I$ is a subset of $C$ and, consequently, of $D$.

THEOREм 37. If $D$ is a pseudo-simple domain, $L$ is an element of a collection $\Delta$ of open curves which surrounds $D$ minimally with respect to a simply connected domain $Q$ and a point $P$, and $O X$ and $O^{\prime} X^{\prime}$ are arcs lying in $S-\bar{D}$ except for their end points $X$ and $X^{\prime}$ which lie on $L$, then the arcs $O X$ and $O^{\prime} X^{\prime}$ abut on $L$ from the same side.

Proof. With the help of Theorems 11(b) and 34 it can be shown that there exists an $\operatorname{arc} F F^{\prime}$ lying in $D$ except for the end points $F$ and $F^{\prime}$, which lie on $L$, such that the interval $F F^{\prime}$ of $L$ contains $X$ and $X^{\prime}$ as interior points. Then by Theorem 36, $J=F F^{\prime}+F F^{\prime}$ (of $L$ ) is a simple closed curved one of whose complementary domains is a subset of $D$. The other complementary domain of $J$ contains the segments $\overparen{O X}$ and $\widehat{O^{\prime}} X^{\prime}$. Hence, the arcs approach $F F^{\prime}$ of $L$ and, therefore, $L$ from the same side.

THEOREM 38. If $D$ is a pseudo-simple domain with respect to a simply connected domain $Q$ and a point $P$, and $O X$ and $O^{\prime} X^{\prime}$ are arcs which have their

$\dagger$ From the definition of a pseudo-simple domain, $Q$ contains no simple domain containing $P$. 
end points $X$ and $X^{\prime}$ in an element $L$ of a collection $\Delta$ of open curves which surrounds $D$, but have no other points in common with the boundary of $D$, and approach $L$ from different sides, then one of these arcs lies in $D$ and the other lies in $S-\bar{D}$.

Theorem 38 is a consequence of Theorems 35 and 37.

THEOREM 39. If $\Delta$ is a collection of open curves which surrounds a pseudosimple domain minimally with respect to a simply connected domain $Q$ and a point $P$, then the boundary of $D$ is $\Delta^{*}$, and every point of $\Delta^{*}$ is accessible from $D$.

Proof. Let $X$ denote a point of an open curve $L$ of $\Delta$, and let $A X B$ denote an interval of $\Delta$ containing $X$ as an interior point. By Theorem 19 there exist two segments, $\overparen{E X}$ and $\widetilde{E^{\prime} X}$, which abut on $A X B$ at $X$ from different sides. The segments, $\overparen{E X}$ and $\overparen{E^{\prime} X}$, contain segments, $\overparen{F X}$ and $\widetilde{F^{\prime} X}$, respectively, which abut on $L$ from different sides and do not contain $P$ or any point of the boundary of $D$ except $X$. By Theorem 38 one of the latter two segments is a subset of $D$.

THEOREM 40. If $X$ is a point of the component $L$ of the boundary $\beta$ of $a$ domain $D$ which is pseudo-simple with respect to a simply connected domain $Q$ and a point $P$, and $M$ is a closed and compact point set not containing $X$, there exists a simple closed curve lying in $S-(D+M)$ whose common part with $\beta$ is an interval of $L$ containing $X$ as an interior point, and whose interior with respect to $P$ contains no point of $M$.

Proof. Let $X P$ denote an arc from $X$ to $P$ lying in $D+X$, and let $J$ denote a simple closed curve separating $X$ from $P$ such that $J \cdot X P$ is a connected subset of $D$. The curve $J$ contains a point of $S-D$. Let $O$ denote the first point that $X P$ has in common with $J$, and let $A O B$ denote the interval of $J$ containing $O$ and lying in $D$ except for its end points $A$ and $B$ which lie on $L$. The interval $A B$ of $L$ contains $X$ because one of the complementary domains of the simple closed curve $A O B+A B$ is a subset of $D$ and since it can not contain $P$, it must contain the segment $\overparen{O X}$ of $X P$. Let $A Z B$ denote the arc of $J$ such that $J=A X B+A Z B$. In the order $A, Z, B$, let $A_{0}$ denote the last point that $A Z B$ has in common with the ray $\overrightarrow{X A}$ of $L$, and let $B_{1}$ denote the next point that $A Z B$ has in common with $\beta$ if it is not in $L$. Let $L_{1}$ denote the component of $\beta$ containing $B_{1}$, and let $A_{1}$ denote the last point that $A Z B$ has in common with $L_{1}$. Let $B_{2}$ denote the next point that $A Z B$ has in common with $\beta$ if it is not in $L$, let $L_{2}$ denote the open curve of $\beta$ containing $B_{2}$, and let $A_{2}$ denote the last point that $A Z B$ has in common with $L_{2}$. This process can be continued (for a finite number of steps) until for some integer $n$ the next point that $A Z B$ has in common with $\beta$ following $A_{n}$ is a point $B_{0}$ of 
$L$. Since $A_{0}$ precedes $B_{0}$ in $A Z B, B_{0}$ belongs to the ray $\overrightarrow{A B}$ of $L$. Let $J_{1}$ denote the simple closed curve $A_{0} X B_{0}$ (of $\left.L\right)+A_{0} B_{1}$ (of $\left.A Z B\right)+A_{1} B_{1}$ (of $\left.L_{1}\right)+A_{1} B_{2}$ (of $A Z B)+\cdots+A_{n-1} B_{n}($ of $A Z B)+A_{n} B_{n}\left(\right.$ of $\left.L_{n}\right)+A_{n} B_{0}$ (of $\left.A Z B\right)$. (See Figure 3.) It is clear that if $B_{1}$ exists, $J_{1}$ lies in $S-D$, because by Theorem 11(b) any

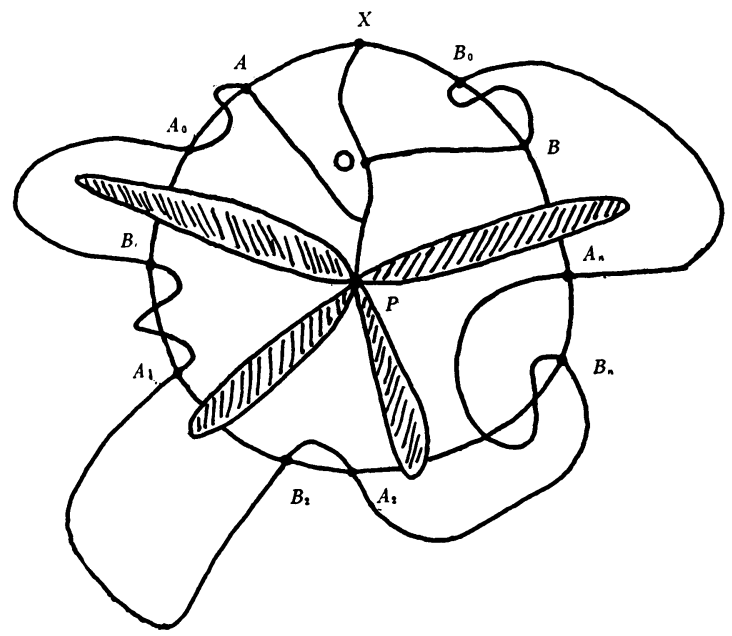

FIGURE 3

segment lying in $D$ having its end points in different components of $\beta$ contains $P$, and $J_{1}$ does not contain $P$. Now in case $J_{1}=A_{0} X B_{0}$ (of $\left.L\right)+A_{0} B_{0}$ (of $A Z B$ ), suppose that $\overparen{A_{0} B_{0}}$ lies in $D$. Then $J_{1}$ lies in $\bar{D}$ and $\beta \cdot J_{1}$ is connected; so by Theorem 36 one of the complementary domains $I$ of $J_{1}$ is a subset of $D$. Let $P^{\prime} X$ denote an arc lying in $I$ except for $X$ which is on the boundary of $I$. Now $I$ does not contain $P$ and $J_{1}$ does not intersect $\overparen{P X}+P$; hence, $I$ does not contain $\overparen{P X}$, and therefore $P X$ and $P^{\prime} X$ abut on $A X B$ from different sides. But since both $\overparen{P X}$ and $P^{\prime} X$ lie in $D$, this contradicts Theorem 35 .

Thus in either case $J_{1}$ is a subset of $S-D$. By Theorem 16 one of the complementary domains $I_{1}$ of $J_{1}$ is a subset of $S-\bar{D}$ and is the interior of $J_{1}$ with respect to $P$. Let $X^{\prime}$ denote a point of $J_{1}-J_{1} \cdot L$, and let $J_{2}$ denote a simple closed curve separating $X$ from $P+M+X^{\prime}$. Let $I_{2}$ denote the interior of $J_{2}$ with respect to $P$. By Theorem 11 of Chapter III of Foundations, $J_{1}+J_{2}$ contains a simple closed curve $J_{3}$ such that (1) $I_{3}$, the interior of $J_{3}$ with respect to $P$, is a subset both of $I_{1}$ and of $I_{2}$, and (2) $J_{3} \cdot L$ is an arc containing $X$ as an interior point. Let $A^{\prime} X^{\prime} B^{\prime}$ denote an arc lying in $I_{3}$ except for points $A^{\prime}$ and $B^{\prime}$ which belong to $J_{3} \cdot \widetilde{A_{0} X}$ and $J_{3} \cdot \widetilde{B_{0} X}$ respectively. Then $J_{4}=A^{\prime} X B^{\prime}$ (of $L)+A^{\prime} X^{\prime} B^{\prime}$ is a simple closed curve lying in $S-(D+M)$ whose interior with respect to $P$ is a subset of $I_{3}$ and hence, contains no point of $M$. Furthermore, 
the common part of $J_{4}$ with $\beta$ is the arc $A^{\prime} X B^{\prime}$ which is an interval of $L$ containing $X$ as an interior point.

THEOREM 41. If $D$ is a pseudo-simple domain, then $S-\bar{D}$ is connected.

Proof. The boundary $\beta$ of $D$ is the sum of a finite number of mutually exclusive open curves $L_{1}, L_{2}, \cdots, L_{j}$. By Theorem 40 there exists, for each point $X$ of $\beta$, a simple closed curve $J_{X}$ such that $J_{X}$ is a subset of $S-D$ and $J_{X} \cdot \beta$ is for some $n \leqq j$ an interval $T_{X}$ of $L_{n}$ containing $X$ as an interior point. Let $I_{X}$ denote the complementary domain of $J_{X}$ which does not contain $D$. By Theorem 16, $I_{X}$ is a subset of $S-\bar{D}$. Now for each $n, n \leqq j$, let $D_{n}=\sum I_{X}$ for $X$ on $L_{n}$. All the segments $J_{X}-T_{X}$ for $X$ on $L_{n}$ lie in $S-\bar{D}$ and abut on $L_{n}$ from the same side. Hence the domains $I_{X}$ overlap, so that $D_{n}$ is a connected subset of $S-\bar{D}$. Furthermore, if $T$ is any arc in $S-D$ which abuts on $L_{n}$ at a point $X$, it abuts on $L_{n}$ from the same side as $J_{X}-T_{X}$, and hence, contains points of $I_{X}$ and $D_{n}$. So for each $n \leqq j$; only one component of $S-\bar{D}$ has boundary points on $L_{n}$.

Now suppose that $S-\bar{D}$ is not connected. Then there exist two mutually exclusive domains $H$ and $K$ such that $S-\bar{D}=H+K$. Since space is connected, both $H$ and $K$ have boundary points in $\beta$, but for no $n, n \leqq j$, does $L_{n}$ contain boundary points of both $H$ and $K$. Hence $\beta \cdot \bar{H}$ and $\beta \cdot \bar{K}$ are mutually exclusive closed point sets. Let $A B$ denote an arc in $S-P$ irreducible from $\beta \cdot \bar{H}$ to $\beta \cdot \bar{K}$. Now since each $D_{n}, n \leqq j$, belongs either to $H$ or to $K$, $\beta=\beta \cdot \bar{H}+\beta \cdot \bar{K}$; so $A B$ contains only two points $A$ and $B$ of $\beta$, and these points belong to different open curves of the collection $L_{1}, L_{2}, \cdots, L_{j}$. Therefore, since $A B$ does not contain $P$, the segment $A B$ lies in $S-\bar{D}$, and hence, contains a point of $I_{A}$ and a point of $I_{B}$. But, since $I_{A}$ and $I_{B}$ belong to $H$ and $K$ respectively, this is a contradiction.

THEOREM 42. Every pseudo-simple domain is strongly simply connected.

This theorem is a consequence of Theorems 16 and 41 .

Definition. A domain $D$ is said to be internally simple provided that for each point $X$ of $D$ there exists a simple domain containing $X$ and lying together with its boundary in $D$.

THEOREM 43. If $D$ is a pseudo-simple domain with respect to a simply connected domain $Q$ and a point $P$, then each component of $D-P$ is internally simple.

Proof. Let $U$ denote a component of $D-P$, and let $X$ denote a point of $U$. Let $A P$ denote an arc from $A$ to $P$ lying except for $P$ in $U$. By Theorems 2 and 10 there exists a simple closed curve $J$ crossing $X P$. If $J$ intersects the boundary of $D$, then $J$ contains an arc $A Y B$ crossing $X P$ and lying except 
for the points $A$ and $B$ of $L$ in $D$. Let $J_{1}$ denote the simple closed curve $A B$ (of $L)+A Y B$. One of the complementary domains of $J_{1}$ is a subset of $D$ and contains $X$. Let $J_{X}$ denote a simple closed curve separating $J_{1}+P$ from $X$. If $J$ does not intersect the boundary of $D$, let $J_{X}$ denote $J$. Then, one of the complementary domains $I_{X}$ of $J_{X}$ contains $X$, and $I_{X}+J_{X}$ is a subset of $U$. Hence, $U$ is internally simple.

THEOREM 44. If $D$ is a pseudo-simple domain, $S-\bar{D}$ is internally simple.

Proof. The domain $D$ is pseudo-simple with respect to a domain $Q$ and a point $P$. Let $\Delta$ denote the collection of mutually exclusive open curves whose sum is the boundary of $D$, and let $L$ denote an element of $\Delta$. Let $X$ denote a point of $S-\bar{D}$, and let $A X$ denote an arc irreducible from $L$ to $X$ and containing no point of $\Delta^{*}-L$. Suppose that $S-(\bar{D}+A X)$ is the sum of two mutually exclusive domains $H$ and $K$. Because no arc separates space, both $H$ and $K$ have boundary points in $\Delta^{*}$. Furthermore, because of Theorem 40 no element of $\Delta-L$ contains points of both $\Delta^{*} \cdot \bar{H}$ and $\Delta^{*} \cdot \bar{K}$, nor does either of the rays of $L$ from $A$ contain points of both $\Delta^{*} \cdot \bar{H}$ and $\Delta^{*} \cdot \bar{K}$. Let $A P$ denote an arc in $D+A$ from $A$ to $P$. Since $A X+A P$ does not separate space, there exists an arc $T$ irreducible from $\Delta^{*} \cdot \bar{H}$ to $\Delta^{*} \cdot \bar{K}$ in $S-(A X+A P)$. Now the segment $\widehat{T}$ is a subset of $S-\bar{D}$, for if $\widehat{T}$ were a subset of $D$, it would have one end on one ray of $L$ from $A$ and the other end on the other ray from $A$ without intersecting $A P$, which by Theorems 35 and 36 is impossible. But it is also impossible for $\widehat{T}$ to lie in $S-\bar{D}$, for in this case $H$ and $K$ would have boundary points in the same open curve of $\Delta$. Hence, $S-(\bar{D}+A X)$ is connected.

Let $E Y F$ denote an arc lying except for $Y$ in $S-(\bar{D}+A X)$ and crossing $A X$ at the point $Y$. Since $S-(\bar{D}+A X)$ is connected, there exists an arc $E Z F$ lying in it from $E$ to $F$. The sum of the arcs, $E Y F$ and $E Z F$, contains a simple closed curve $J$ crossing $A X$ at the point $Y$ whose interior with respect to $P$ is a simple domain $I$ containing $X$ such that $I+J$ is a subset of $S-\bar{D}$.

THEOREM 45. If $D_{1}$ and $D_{2}$ are pseudo-simple domains with respect to domains $Q_{1}$ and $Q_{2}$ and a point $P$ respectively, $Q_{2}$ is a subset of $D_{1}$, and $C$ is a component of $D_{1}-\bar{D}_{2}$, then $C$ is an internally simple, strongly simply connected domain.

Proof. The component $C$ is strongly simply connected; for if a simple closed curve $J$ is a subset of $\bar{C}$, then by Theorems 16 and 41 the interior $I$ of $J$ with respect to $P$ is a subset of both $D_{1}$ and $S-\bar{D}_{2}$.

If $X$ is a point of $C$, then by Theorems 43 and 44 there exist two simple domains, $I_{1}$ and $I_{2}$, each containing $X$ such that $\bar{I}_{1}$ and $\bar{I}_{2}$ are subsets of $D_{1}-P$ and $S-\bar{D}_{2}$ respectively. By Theorem 12 of Chapter III of Foundations, $I_{1} \cdot I_{2}$ 
contains a simple domain $I_{X}$ which contains $X$ and whose boundary is a subset of the sum of the boundaries of $I_{1}$ and $I_{2} \cdot \dagger$ Therefore, $\bar{I}_{X}$ is a subset of $C$, and $C$ is internally simple.

THEOREM 46. If $D$ is an internally simple, simply connected domain and $A B$ is an arc lying in $D$ except for its end points which belong to the boundary of $D$, then $D-D \cdot A B$ is the sum of two mutually exclusive, internally simple, simply connected domains, each having $A B$ on its boundary.

Proof. For each point $X$ of $\overparen{A B}$ let $I_{X}$ denote a simple domain containing $X$ such that $\bar{I}_{X}$ is a subset of $D$. Let $J_{X}$ denote the boundary of $I_{X}$ and let $C$ denote a simple closed curve separating $X$ from $A+B+J_{X}$. By Theorem 10, $C+\overparen{A B}$ contains a simple closed curve $C^{\prime}$ separating $X$ from $A+B+J_{X}$, whose common part with $A B$ is the sum of two continua. Let $A^{\prime} X B^{\prime}$ denote the interval of $A B$ containing $X$ such that $A^{\prime} X B^{\prime} \cdot C^{\prime}=A^{\prime}+B^{\prime}$. One of the complementary domains $I^{\prime}$ of $C^{\prime}$ is a subset of $D$ and contains the segment $A^{\prime} X B^{\prime}$. The point set $I^{\prime}-\widehat{A^{\prime} X B^{\prime}}$ is the sum of two simple domains $I_{X 1}$ and $I_{X 2}$, one on one side of $A B$ and the other on the other side of $A B$. Now if the subscripts 1 and 2 are so used so that all the domains $I_{X 1}$ for $X$ on $A B$ are on the same side of $A B$ and all the domains $I_{X 2}$ are on the other side of $A B$, $D_{1}=\sum I_{X 1}$ and $D_{2}=\sum I_{X 2}$ are two connected domains which are subsets of $D$. Now each point $O$ of $D-\overparen{A B}$ may be joined to a point of $\overparen{A B}$ by an arc $O E$ lying in $D-\overparen{A B}$ except for its one end point $E$, and $\overparen{O E}$ must intersect either $D_{1}$ or $D_{2}$. If for each $i, i=1,2, H_{i}$ denotes the set of all points $O$ such that the segment $\overparen{O E}$ intersects $D_{i}$, then $H_{1}$ and $H_{2}$ are connected domains and $H_{1}+H_{2}=D-\overparen{A B}$. But if $D$ is simply connected, no arc segment lying in $D$ can abut on $A B$ from different sides since neither $A$ nor $B$ belongs to $D$. Hence $H_{1}$ and $H_{2}$ are mutually exclusive, and $A B$ belongs to the boundary of each of them.

Now suppose that $J$ is a simple closed curve lying in $H_{1}$. One of the complementary domains $I$ of $J$ is a subset of $D$. Therefore, $I$ contains no point of $A B$. But $I$ has limit points in $H_{1}$, namely, the points of $J$, and hence contains points of $H_{1}$. Consequently $I$ is a subset of $H_{1}$, and therefore $H_{1}$ is simply connected. Likewise, $H_{2}$ is simply connected.

Let $X$ denote a point of $H_{1}$. Since $D$ is internally simple, there exists a simple domain $I_{X}^{\prime}$ containing $X$ and lying together with its boundary $J_{X}^{\prime}$ in $D$. By Theorem 3 there exists a simple closed curve $J_{X}$ separating $X$ from $J_{X}^{\prime}+A B$. The curve $J_{X}$ is a subset of $H_{1}$, and hence one of its complementary domains $I_{X}$ is a subset of $H_{1}$ and contains $X$. Consequently, $H_{1}$ is internally simple and likewise $\mathrm{H}_{2}$ is internally simple.

$\dagger$ The notation here does not correspond to that of Theorem 12 of Chapter III of Foundations. 
THEOREM 47. If $D$ is an internally simple, simply connected domain, $\beta$ is the boundary of $D, J$ is a simple closed curve lying in $D$, and $A B$ is an arc irreducible from $J$ to the boundary of $D$, then $D-(J+\overparen{A B})$ is the sum of two mutually exclusive, internally simple, simply connected domains, one having $J$ for its boundary, and the other having for its boundary $J+A B+\beta$.

Theorem 47 may be established by methods similar to those used in proving Theorem 46.

THEOREM 48. If $D$ is a pseudo-simple domain and $A B$ is an arc lying in $S-\bar{D}$ except for the points $A$ and $B$ which lie on the boundary of $D$, then $S-(\bar{D}+A B)$ is the sum of two connected domains whose boundaries have as their common part the arc $A B$.

Proof. From Theorems $16,41,44$, and $46, S-(\bar{D}+A B)$ is the sum of two connected domains whose boundaries contain $A B$ in their common part. And if $\beta$ denotes the boundary of $D$, it is clear from Theorems 38 and 40 that this common part cannot contain a point of $\beta-(A+B)$.

Theorem 49. If under the hypothesis of Theorem 45, $A X B$ is an arc lying in $C$ except for the points $A$ and $B$ which lie on the boundary of $C$, then $C-\widehat{A X B}$ is the sum of two connected domains whose boundaries have as their common part the $\operatorname{arc} A X B$.

Theorem 49 may be proved by an argument similar to that of the preceding theorem if we use Theorems 45 and 46 and a theorem similar to Theorem 40 and established in a similar manner.

Definition. Suppose that $X_{1}, X_{2}, X_{3}$, and $X_{4}$ are points of $\beta$, the boundary of a pseudo-simple domain. Then $X_{1}+X_{3}$ is said to ordinally separate $X_{2}$ from $X_{4}$ on $\beta$ if there exists a simple closed curve $J$ whose common part with $\beta$ is $X_{1}+X_{3}$ and which separates $X_{2}$ from $X_{4}$.

Theorem 50. Suppose that $D$ is a pseudo-simple domain, $\beta$ is the boundary of $D$, and $X_{1}, X_{2}, X_{3}$, and $X_{4}$ are four distinct points of $\beta$. Then one pair of them ordinally separates the other two on $\beta$; and if $X_{1}+X_{3}$ ordinally separates $X_{2}$ from $X_{4}$ on $\beta$, then (1) $X_{2}+X_{4}$ ordinally separates $X_{1}$ from $X_{3}$ on $\beta$, (2) every simple closed curve $J$ containing a point of $D$ and $S-D$ such that $J \cdot \beta=X_{1}+X_{3}$ separates $X_{2}$ from $X_{4}$, but (3) $X_{1}+X_{2}$ does not ordinally separate $X_{3}$ from $X_{4}$ on $\beta$.

Proof. Let $X_{1} A X_{2}$ and $X_{1} B X_{2}$ denote arcs lying except for $X_{1}$ and $X_{2}$ in $D$ and $S-\bar{D}$ respectively. Suppose that $J^{\prime}=X_{1} A X_{2}+X_{1} B X_{2}$ does not separate $X_{3}$ from $X_{4}$. Now let $A X_{3}$ and $B X_{3}$ denote arcs lying except for $X_{3}$ in $D$ and $S-\bar{D}$ respectively, such that $A X_{3} \cdot J^{\prime}=A$ and $B X_{3} \cdot J^{\prime}=B$. With respect to $X_{4}$, the interior of the simple closed curves formed by one pair of the three $\operatorname{arcs} A X_{1} B, A X_{2} B$, and $A X_{3} B$ contains the other arc segment. Hence, one 
pair of the points $X_{1}, X_{2}, X_{3}$, and $X_{4}$ ordinally separates the other two on $\beta$.

Suppose that $X_{1}+X_{3}$ ordinally separates $X_{2}$ from $X_{4}$ on $\beta$. Then there exists by a construction similar to that of the above paragraph two arcs $X_{2} A X_{4}$ and $X_{2} B X_{4}$ lying except for $X_{2}$ and $X_{4}$ in $D$ and $S-\bar{D}$ respectively whose sum is a simple closed curve separating $X_{1}$ from $X_{3}$. If $J$ is a simple closed curve containing a point of $D$ and a point of $S-\bar{D}$ whose common part with $\beta$ is $X_{1}+X_{3}$, then $J$ is the sum of two $\operatorname{arcs} X_{1} A^{\prime} X_{3}$ and $X_{1} B^{\prime} X_{3}$ lying except for $X_{1}$ and $X_{3}$ in $D$ and $S-\bar{D}$ respectively. Let $C$ denote the simple closed curve $X_{2} A X_{4}+X_{2} B X_{4}$. By a double application of Theorem 10, $C+{\widehat{X_{1} A^{\prime} X_{3}}}_{3}+{\widehat{X_{1} B^{\prime} X_{3}}}_{3}$ contains a simple closed curve $C^{\prime}$ whose common part with ${\widehat{X_{1} A^{\prime} X_{3}}}_{3}$ is connected and whose common part with ${\widehat{X_{1} B^{\prime} X_{3}}}_{3}$ is also connected and which separates $X_{2}$ from $X_{4}$. It is clear now that $J$ separates $X_{2}$ from $X_{4}$.

Now suppose that $X_{1}+X_{2}$ ordinally separates $X_{3}$ from $X_{4}$ on $\beta$. Then there would exist a simple closed curve, having the properties of $J$ in the preceding paragraph, which does not separate $X_{2}$ from $X_{4}$. This is impossible if $X_{1}+X_{3}$ ordinally separates $X_{2}$ from $X_{4}$.

Arguments of a similar nature show that if order on $\beta$ is interpreted by the notion of ordinal separation, then we have

THEOREM 51. If $\beta$ is the boundary of a pseudo-simple domain, the points of $\beta$ have a cyclic order which preserves the ordinary order on any open curve component of $\beta$.

Theorem 52. Suppose that $D_{1}$ and $D_{2}$ are pseudo-simple domains with respect to domains $Q_{1}$ and $Q_{2}$ and a point $P$ respectively, $Q_{2}$ is a subset of $D_{1}, X_{1}$, $X_{2}, X_{3}$, and $X_{4}$, and $Y_{1}, Y_{2}, Y_{3}$, and $Y_{4}$ are the end points of four mutually exclusive arcs $T_{1}, T_{2}, T_{3}$, and $T_{4}$ which cross $D_{1}-\bar{D}_{2}$. Then if $X_{1}+X_{3}$ ordinally separates $X_{2}$ from $X_{4}$ on $\beta_{1}$, the boundary of $D_{1}$, then $Y_{1}+Y_{2}$ ordinally separates $Y_{2}$ from $Y_{4}$ on $\beta_{2}$, the boundary of $D_{2}$, and conversely.

Proof. Let $X_{1} A X_{2}$ denote an arc lying except for the points $X_{1}$ and $X_{3}$ in $S-\bar{D}_{1}$, and let $Y_{1} B Y_{3}$ denote an arc lying except for the points $Y_{1}$ and $Y_{3}$ in $D_{2}$. Let $J$ denote the simple closed curve $T_{1}+X_{1} A X_{3}+T_{3}+Y_{1} B Y_{3}$. Since $X_{1}+X_{3}$ ordinally separates $X_{2}$ from $X_{4}$ on $\beta_{1}, J$ separates $X_{2}$ from $X_{4}$. But $J$ does not intersect $T_{2}$ or $T_{4}$ and hence, $J$ separates $Y_{2}$ from $Y_{4}$. Therefore, $Y_{1}+Y_{2}$ ordinally separates $Y_{2}$ from $Y_{4}$ on $\beta_{2}$. Likewise, the converse is true.

2. Consequences of Axioms $0-4,5_{1}^{*}$ AND 7

Ахгом 7. The set of all points is completely separable. $\dagger$

$\dagger$ This axiom is numbered 7 because it is so numbered in Foundations. See Foundations, p. 412. 
The purpose of this section is to outline an argument showing that a space satisfying Axioms $0-4,5_{1}^{*}$, and 7 is a subset of a space satisfying Axioms $0-4,5_{1}^{*}$, and 7 which contains no edge points. Considerable detail will be left to the reader, and notation introduced in a definition, when used subsequently without explanation, will be understood to have the significance given it in the definition.

Definition. Suppose that $P$ is an edge point. Suppose, furthermore, that there exist for each $n$, a pseudo-simple domain $D_{n}$ containing $\bar{D}_{n+1}$ whose boundary $\beta_{n}$ is the sum of a finite number of open curves, $L_{n 1}, L_{n 2}, \cdots, L_{n q_{n}}$, and for each $n$ and $q, q \leqq q_{n}$, two sequences of mutually exclusive arcs, $T_{1, n q}, T_{2, n q}, \cdots$ and $T_{-1, n q}, T_{-2, n q}, \cdots$, crossing $D_{n}-\bar{D}_{n+1}$ such that, denoting $T_{m, n q} \cdot \beta_{n}$ and $T_{m, n q} \cdot \beta_{n+1}$ by $X_{m, n q}$ and $Y_{m, n q}$ respectively, $\dagger$

(0) for each $n, D_{n}$ is pseudo-simple with respect to $P$ and a domain $Q_{n}$ which is a subset of a region of $G_{n}$ of Axiom 1, which is in turn a subset of $D_{n-1}, D_{0}=S$;

(1) for each $m, L_{n q}$ contains $X_{m, n q}$;

(2) for each $n$, the points $X_{1, n 1}, X_{2, n 1}, \cdots, \cdots, X_{-2, n 2}, X_{-1, n 2}, X_{1, n 2}$, $X_{2, n 2}, \cdots, \cdots, \cdots, X_{-2, n q_{n}}, X_{-1, n q_{n}}, X_{1, n q_{n}}, X_{2, n q_{n}}, \cdots, \cdots, X_{-2, n 1}$, $X_{-1, n 1}$ have on $\beta_{n}$ the cyclic order indicated; $\ddagger$

(3) for each $Y_{m, n q}$, there exists an integer $r, r \geqq q$, such that $X_{m,(n+1) r}$ $=Y_{m, n q}$;

(4) for each $n$ and $q, q \leqq q_{n}$, neither of the sequences, $T_{1, n q}, T_{2, n q}, \cdots$ and $T_{-1, n q}, T_{-2, n q}, \cdots$ has a sequential limit set. Let $W s$ denote the point set consisting of $P$ together with all the sets $L_{n q}$ and $T_{m, n q}$. Such a set, $W s$, is said to be a radial web skeleton about $P$.

THEOREM 53. If $R$ is a region containing an edge point $P, R$ contains a radial web skeleton about $P$.

Theorem 53 may be proved with the help of Theorems 15, 18, 32, 33, 45, and 52 and a method similar to that of Theorem 33.

Definition AND Notation. Suppose that $W s$ is a radial web skeleton about an edge point $P$. For each $n$ and $q, q \leqq q_{n}, L_{n q}$ of $W s$ is the sum of two rays $L_{n q}^{+}$and $L_{n q}^{-}$having only one point in common and containing $X_{1, n q}$, $X_{2, n q}, \cdots$ and $X_{-1, n q}, X_{-2, n q}, \cdots$ respectively. Such rays are said to be rays on $\beta_{n}$ and to have the cyclic order of the points $X_{m, n q}$. The point set $W s$ is said to connect $L_{n q}^{+}$to $L_{(n+1) r}^{+}$if $T_{1, n q}$ contains points of both of them. Likewise, $W s$ is said to connect $L_{n q}^{-}$to $L_{(n+1) r}^{-}$if $T_{-1, n q}$ contains points of both of them.

$\dagger$ When an integral subscript letter is followed by a comma, its range is both the positive and negative integers.

$\ddagger$ This order is selected arbitrarily on $\beta_{1}$; then it is transferred from $\beta_{1}$ to $\beta_{2}$, from $\beta_{2}$, to $\beta_{3}, \cdots$ by the $\operatorname{arcs} T_{m, n q}$ using Theorem 52 . 
ThEOREM 54. Suppose that $W$ s is a radial web skeleton about an edge point $P$, and Ws connects $L_{n q}^{+}$to $L_{(n+1) r}^{+}$and $\mathrm{L}_{n q^{\prime}}^{-}$to $L_{(n+1) r^{\prime}}^{-}$. Then if $q^{\prime}=q+1, r^{\prime}=r+1$, and conversely.

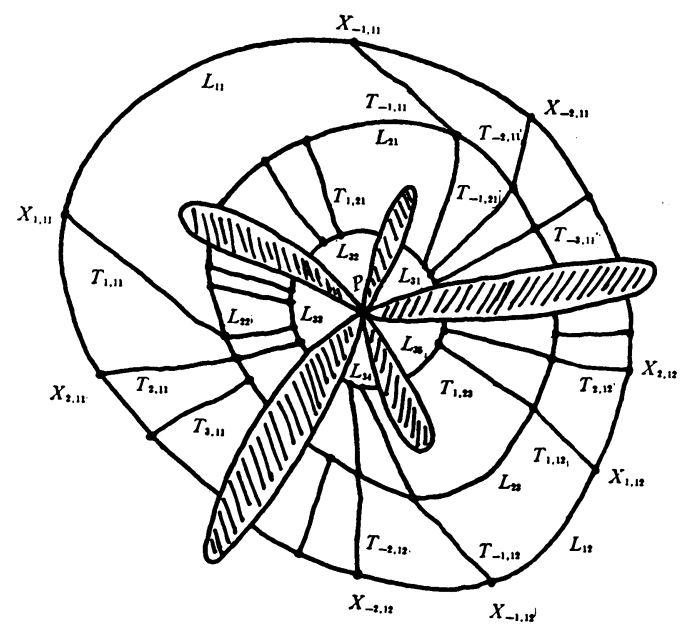

FIGURE 4

Proof. Suppose on the contrary, for instance, that $T_{1,11}$ contains a point of $L_{11}^{+}$and of $L_{23}^{+}$while $T_{-1,12}$ contains a point of $L_{12}^{-}$and of $L_{25}^{-}$. Since $T_{1,11}+T_{-1,12}+P$ does not separate space, there exists an $\operatorname{arc} T$ from $L_{24}$ to $\beta_{1}$ of $W s$ lying except for its end points in $D_{1}-\bar{D}_{2}$. Let $T \cdot \beta_{1}=X$ and $T \cdot \beta_{2}=Y$. Since $Y$ is between $Y_{1,11}$ and $Y_{-1,12}$ on $\beta_{2}, X$ is between $X_{1,11}$ and $X_{-1,12}$ on $\beta_{1}$ by Theorem 52 . Hence $X$ is either on the subray of $L_{11}^{+}$from $X_{1,11}$ or on the subray of $L_{12}^{-}$from $X_{-1,12}$. Suppose the former, and a similar argument will apply to the other case. Since the sequence of $\operatorname{arcs} T_{1,11}, T_{2,11}, T_{3,11}, \cdots$ has no sequential limit set, there is some one of them, $T_{m, 11}$, which has no point in common with $T$ such that $X_{m, 11}$ is between $X$ and $X_{-1,12}$ on $\beta_{1}$. But $Y_{m, 11}$ belongs to $L_{23}^{+}$and is not between $Y$ and $Y_{-1,12}$ on $\beta_{2}$, which contradicts Theorem 52.

A similar argument will show the converse to be true; that is, if $r^{\prime}=r+1$, then $q^{\prime}=q+1$.

Definition. Suppose that $W s$ is a radial web skeleton. Then $L_{n q}^{+}$and $L_{n(q+1)}^{-}$of $W s$ are said to be adjacent rays on $\beta_{n} . \dagger$

THEOREM 55. Suppose that $W$ s is a radial web skeleton. Then $W s$ connects each pair of adjacent rays on $\beta_{n}$ to a pair of adjacent rays on $\beta_{n+1}$, and if $W s$

$\dagger$ When $q=q_{n}, q+1$ is to be interpreted as 1 . 
connects either of a pair of adjacent rays on $\beta_{n}$ to a ray on $\beta_{n-1}$, then $W$ s connects this pair to a pair of adjacent rays on $\beta_{n-1}$.

Notation. If $W s$ is a radial web skeleton, let $C_{n q}$ denote the component of $D_{n}-\bar{D}_{n+1}$ whose boundary $\beta_{n q}$ contains $L_{n q}$ of $W s$.

THEOREM 56. If $W$ s is a radial web skeleton, then $\beta_{n q} \cdot \beta_{n+1}$ is the sum of a finite collection $H_{n q}$ of consecutive rays on $\beta_{n+1}$ consisting of two extreme rays, $L_{(n+1) q}^{-}$and $L_{(n+1) q^{\prime}}^{+}, q \leqq q^{\prime}$, and all other rays on $\beta_{n+1}$ between $L_{(n+1) q}^{-}$and $L_{(n+1) q^{\prime}}^{+}$; and $L_{(n+1) q}^{-}$and $L_{(n+1) q^{\prime}}^{+}$are the only rays of $H_{n q}$ which are connected by $W$ s to rays of $\beta_{n}$.

THEOREM 57. If $W s$ is a radial web skeleton and $n$ and $q$ are integers such that neither $L_{n q}^{+}$nor $L_{n(q+1)}^{-}$of $W$ s is connected to a ray on $\beta_{n-1}$ by $W s$, then there exists a sequence of mutually exclusive arcs $U_{1[n q]}, U_{2[n q]}, U_{3[n q]}, \cdots \dagger$ such that (1) for each $m, m>0, U_{m[n q]}$ is an arc from $X_{m, n q}$ to $X_{-m, n(q+1)}$ lying except for these two points in $D_{n-1}-\bar{D}_{n}, D_{0}=S$, and (2) the sequence has no sequential limit set.

Proof. Let $C$ denote the component of $D_{n-1}-\bar{D}_{n}$ whose boundary contains $L_{n q}^{+}+L_{n(q+1)}^{-}$, and let $L$ denote $\bar{C} \cdot \beta_{n-1} . \ddagger$ Let $G$ denote the collection of all simple domains which lie together with their boundaries in $C$. By Theorems 44 and $45, G$ covers $C$. Since space is completely separable, $G$ contains a countable subcollection $I_{1}, I_{2}, I_{3}, \cdots$ covering $C$. For each integer $m, m>0$, let $M_{m}$ denote an arc irreducible from $J_{m}$, the boundary of $I_{m}$, to $L$ lying except for one end point in $C$. By Theorem $47, C-C \cdot\left(J_{m}+M_{m}\right)$ is the sum of two connected domains, $I_{m}$ and $E_{m}$, where the boundary of $E_{m}$ consists of $J_{m}+M_{m}$ together with the boundary of $C$. For each $m$, let $U_{m[n q]}^{\prime}$ denote an arc from $X_{m, n q}$ to $X_{-m, n(q+1)}$ lying except for these end points in $E_{m}$. By Theorem $49, U_{m[n q]}^{\prime}$ separates $C$ into two internally simple, simply connected domains. One of these domains $V_{m}^{\prime}$ has for its boundary $U_{m[n q]}^{\prime}$ plus the subray of $L_{n q}^{+}$ from $X_{m, n q}$ and the subray of $L_{n(q+1)}^{-}$from $X_{-m, n(q+1)}$, and contains no point of $I_{m}$. If the arcs $U_{m[n q]}^{\prime}$ are mutually exclusive, then they form the sequence $U_{1[n q]}, U_{2[n q]}, U_{3[n q]}, \cdots$. If this is not the case, let $U_{1[n q]}=U_{1[n q]}^{\prime}$ and $V_{1}=V_{1}^{\prime}$, and suppose that $U_{1[n q]} \cdot U_{2[n q]}^{\prime}$ is not vacuous. Let $A_{1} B_{1}$ and $A_{2} B_{2}$ denote intervals of $U_{1[n q]}$ and $U_{2[n q]}^{\prime}$ respectively which lie in $C$ and contain $U_{1[n q]} \cdot U_{2[n q]}^{\prime}$. By Theorems 10 and 45 and Theorem 13 of Chapter III of Foundations, there exists a simple closed curve $J$ lying in $C$ and separating $A_{1} B_{1}+A_{2} B_{2}$ from $X_{2, n q}+X_{-2, n(q+1)}$ such that the common part of $J$ with each of the intervals $A_{2} X_{2, n q}$ and $B_{2} X_{-2, n(q+1)}$ of $U_{2[n q]}^{\prime}$ is connected. Now since

$\dagger$ This notation, $[n q]$, is used to restrict $n$ and $q$ to values for which the hypothesis of Theorem 57 is satisfied.

$\ddagger$ When $n=1, \bar{C} \cdot \beta_{n-1}$ is to be interpreted as a point of $L_{n q}^{-}$. 
${\widehat{A_{2} X_{2, n q}}}_{\text {and }}{\widehat{B_{2} X}}_{-2, n(q+1)}$ are subsets of $V_{1}$ and contain no points of $V_{2}^{\prime}, J$ contains an arc $A B$ lying in $V_{1} \cdot V_{2}^{\prime}$ except for $A$ and $B$ irreducible from $A_{2} X_{2, n q}$ to $B_{2} X_{-2, n(q+1)}$. Let $U_{2[n q]}=A B+A X_{2, n q}$ of $A_{2} X_{2, n q}+B X_{-2, n(q+1)}$ of $B_{2} X_{-2, n(q+1)}$, and let $V_{2}$ denote the component of $V_{1}-\widehat{U}_{2[n q]}$ which has the subray of $L_{n q}^{+}$ from $X_{2, n q}$ and the subray of $L_{n(q+1)}^{-}$from $X_{-2, n(q+1)}$ on its boundary. The domain $V_{2}$ is a subset of $V_{1} \cdot V_{2}^{\prime}$. This process may be continued and the required sequence of arcs constructed, for it is clear that since $V_{1} \supset V_{2} \supset V_{3} \supset \cdots$ and $\bar{V}_{1} \cdot \bar{V}_{2} \cdot \bar{V}_{3} \ldots=0$, the sequence of arcs has no sequential limit set.

Notation and construction. Now on a radial web skeleton it is possible to construct what will be called a radial web. Let $W s$ denote a radial web skeleton about an edge point $P$. For each integer triplet, $m$ (positive or negative), $n>0$, and $0<q \leqq q_{n}$, there exists a continuous reversible transformation of the point set $T_{m, n q}-Y_{m, n q}$ of $W s$ into the number set $1 / n \geqq t>1 /(n+1)$.

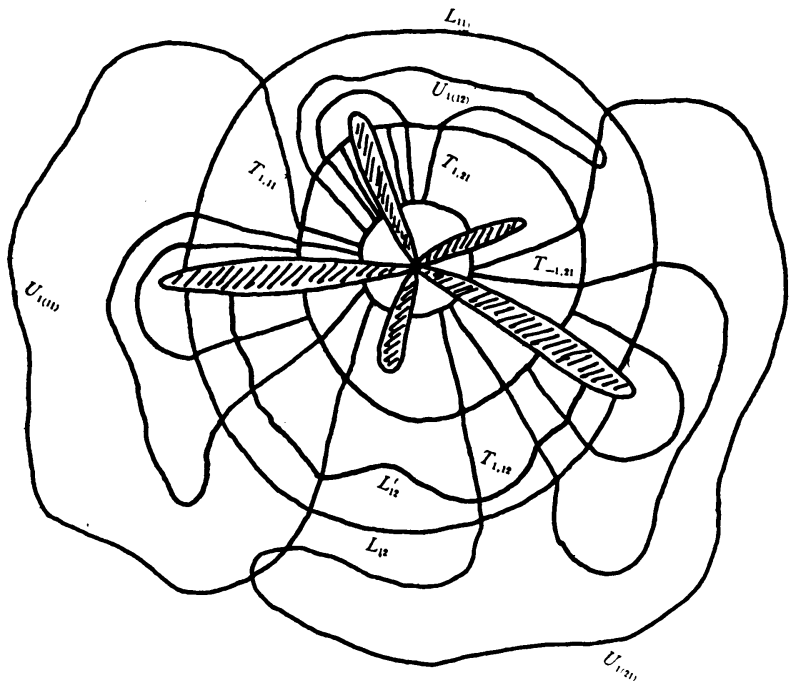

FIGURE 5

Let $X_{m, n q}^{t}$ denote the point of $T_{m, n q}$ whose transform is the number $t$. Then, of course, $X_{m, n q}=X_{m, n q}^{1 / n}$. Let $\rho$ denote a countable set of numbers everywhere dense on $0<t<1$ and containing all the reciprocals of positive integers except 1 . For each pair of positive integers, $n$ and $q, q \leqq q_{n}$, and for each $t$ of $\rho$ such that $1 / n \geqq t>1 /(n+1)$, let $L_{n q}^{t}$ denote an open curve lying in $C_{n q}$ such that (1) $L_{n q}^{t}=L_{n q}$ if $t=1 / n$, (2) $L_{n q}^{t}$ intersects $T_{m, n q}$ in the point $X_{m, n q}^{t}$, and (3) if $t$ and $t^{\prime}$ are different numbers of $\rho, L_{n q}^{t} \cdot L_{n q}^{t^{\prime}}=0$. Since $\rho$ is countable, these open curves may be constructed one at a time by the use of Theorem 18 and repeated applications of Theorem 46.

For each pair of integers, $n$ and $q, n>0$ and $q \leqq q_{n}$, such that $L_{n q}^{+}$is not 
connected by $W s$ to a ray on $\beta_{n-1}$, there exists by Theorem 57 a sequence $U_{1[n q]}, U_{2[n q]}, U_{3[n q]}, \cdots$ of mutually exclusive arcs such that (1) for each $m$, $m>0, U_{m[n q]}$ is an arc from $X_{m, n q}$ to $X_{-m, n(q+1)}$ lying except for these points in $D_{n-1}-\bar{D}_{n}, D_{0}=S$, and (2) the sequence has no sequential limit set. For each $t$ of $\rho$, let $\beta^{t}=\sum_{q=1}^{q_{n}} L_{n q}^{t}$. The point set $W$ consisting of $W s$ together with all the point sets $\beta^{t}$ and $U_{m[n q]}$ is called a radial web about $P$, and $W s$ is said to be the skeleton of $W$.

Notation and definition. If $X_{m, n q}$ is a point of a ray on $\beta_{n}$ of the skeleton $W s$ of the radial web $W$ which is not connected to a ray on $\beta_{n-1}$ by $W s$, let $N_{m,[n q]}$ denote $P+\sum_{r=n}^{\infty} T_{m, r p_{r}}$, where $p_{n}=q$ and for $r>n, p_{r}$ is the integer such that $X_{m, r p_{r}}=Y_{m,(r-1) p_{r-1}}$. For each $[n q]$ and $m, m>0$, let $J_{m[n q]}$ denote the simple closed curve $N_{m,[n q]}+U_{m[n q]}+N_{-m,[n(q+1)]}$, and let $I_{m[n q]}$ denote the complementary domain of $J_{m[n q]}$ which contains $J_{m+1[n q]}-P$. † For each $t$ of $\rho$, let $U_{m[n q]}^{t}$ denote the interval of $J_{m[n q]}-P$ which is irreducible from one component of $\beta^{t}$ to another component of $\beta^{t}$ with $t \leqq 1 / n$.

For each pair of values, $t^{\prime}$ and $t^{\prime \prime}$ of $\rho, t^{\prime}>t^{\prime \prime}$, let $\beta_{m,[n q]}^{t^{\prime} t^{\prime \prime}}$ denote the point set consisting of the interval of $N_{m,[n q]}$ between $\beta^{t^{\prime}}$ and $\beta^{t^{\prime \prime}}$, when both $t^{\prime}$ and $t^{\prime \prime}$ are less than $1 / n$, together with the rays of $\beta^{t^{\prime}}$ and $\beta^{t^{\prime \prime}}$ from the end points of this interval which intersect $N_{m+1,[n q]}$. It is clear that $\beta_{m,[n q]}^{t^{\prime \prime}{ }^{\prime \prime}}$ separates space into two mutually exclusive connected domains, one of which, $I_{m,\left[n q^{\prime}\right]}^{t^{\prime} t^{\prime}}$, is a subset of $I_{m[n q]}$, is the sum of an infinite number of simple domains, and has $\beta_{m,[n q]}^{t^{\prime \prime} t^{\prime \prime}}$ for its complete boundary. For each $m$,

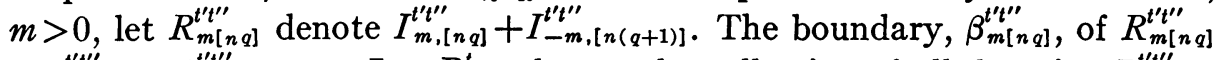
is $\beta_{m,[n q]}^{t^{\prime} t^{\prime \prime}}+\beta_{-m,[n(q+1)]}^{t^{\prime} t^{\prime \prime}}$. Let $P_{[n q]}^{t}$ denote the collection of all domains $R_{m[n q]}^{t^{\prime} t^{\prime \prime}}$, where $t^{\prime \prime}<t<t^{\prime}$. The collection $P_{[n q]}^{t}$ will be hereinafter referred to as an ideal point. $\ddagger$ The set of ideal points, $\sum_{t} P_{[n q]}^{t}$, will be called the spur $N_{[n q]}$ and $\sum N_{[n q]}$ will be called the cluster at $P$ or about $P$.

Theorem 58. For each $[n q]$, the sequence $J_{1[n q]}, J_{2[n q]}, J_{3[n q]}, \cdots$ has $P$ for its sequential limit set and $I_{1[n q]} \cdot I_{2[n q]} \cdot I_{3[n q]}, \cdots=0$.

THEOREM 59. For each $m, W-W \cdot \sum_{[n q]} I_{m[n q]}$ is a closed and compact point set.

Theorem 60. If $P_{[n q]}^{t}$ is an ideal point and $M$ is a compact point set, there exists a number $\delta$ and an integer $\bar{m}$, such that if $0<t^{\prime}-t^{\prime \prime}<\delta, m>\bar{m}$, and $R_{m[n q]}^{t^{\prime} t^{\prime \prime}}$ belongs to $P_{[n q]}^{t}$, then $\bar{R}_{m[n q]}^{t^{\prime} t^{\prime \prime}}$ contains no point of $M$.

THEOREM 61. If $t$ is a number of $\rho$ and $M$ is a compact point set, there exists a number $\bar{m}$ such that if $m>\bar{m}$, then for no $[n q]$ does $U_{m[n q]}^{t}$ contain a point of $M$.

$\dagger$ The point sets $U_{m[n q]}$ may intersect the sets $\beta^{t}$ in more than one point.

$\ddagger$ This is analogous to a method used by R. L. Moore to define point in terms of piece. See R. L. Moore, $A$ set of axioms for plane analysis situs, Fundamenta Mathematicae, vol. 25 (1935), pp. 13-28. 
Notation and construction. By Theorem 24 the set of edge points may be put into a simple countable sequence $P_{1}, P_{2}, P_{3}, \cdots$. Let $W_{1}, W_{2}, W_{3}, \cdots$ and, for each integer $j, R_{1 j}, R_{2 j}, R_{3 j}, \cdots$ and $D_{1 j}, D_{2 j}, D_{3 j}, \cdots$ denote sequences of point sets such that (1) for each $n$ and $j, R_{n j}$ is a region of $G_{n+j}$ of Axiom 1, (2) for each $n$ and $j, D_{n j}$ is a pseudo-simple domain with respect to $P_{j}$ and a simply connected domain $Q_{n j}$ lying with its boundary in $R_{n j}$, and $D_{n j}$ contains $R_{(n+1) j}$, (3) for each $j, W_{j}$ is a radial web about $P_{j}$ whose skeleton is constructed on the domains, $D_{1 j}, D_{2 j}, D_{3 j}, \cdots$, and lies in $R_{1 j}$, (4) if $j^{\prime}>j$ and $R_{1 j^{\prime}}$ contains a point of $D_{1 j}$, then $\bar{R}_{1 j^{\prime}}$ is a subset of $R_{1 j}$, and (5) for each integer $j^{\prime}$, there exists an integer $m$ such that if $j<j^{\prime}$, then $\bar{R}_{1 j^{\prime}}$ contains no point of $\overline{\sum_{[n q]} I_{m[n q]}}$ of $W_{j}$. Let $G$ denote a countable collection of simple domains such that if $X$ is a simple point of $S$ and $R$ is a region containing $X$, some domain of $G$ contains $X$ and lies together with its boundary in $R$. For each integer $i$, let $H_{i}$ denote the collection of all the domains $D$ such that either $\left(1^{\prime}\right) D$ is a simple domain of $G$ which is a subset of a region of $G_{i}$ of Axiom 1 , or $\left(2^{\prime}\right)$ for some pair of integers $n$ and $j, n+j \geqq i, D$ is a pseudosimple domain $D_{n j}$, or $\left(3^{\prime}\right)$ for some triplet of integers, $m, n$, and $q$, and some pair of numbers, $t^{\prime}$ and $t^{\prime \prime}$, of $\rho$, with $m \geqq i$ and $0<t^{\prime}-t^{\prime \prime} \leqq 1 / i, D=R_{m[n q]}^{t^{\prime \prime} t^{\prime \prime}}$ of $W_{j}$ for some $j$.

Definition. If for some $i$ and $j, D$ is a domain of $H_{i}$ and $P_{[n q]}^{t}$ is an ideal point defined from $W_{j}$, then $D$ is said to inclose $P_{[n q]}^{t}$ provided that some domain of $P_{[n q]}^{t}$ together with its boundary is a subset of $D$.

Definition. Let $S^{\prime}$ be a space in which point is to be interpreted as meaning either a point of $S$ or one of the ideal points defined by $W_{j}$ for some $j$, and in which region is to be interpreted as meaning a point set $g^{\prime}$ consisting of the points of some domain $D$ of $H_{i}$, for some $i$, together with the ideal points which $D$ incloses. A point of $S^{\prime}$ which is also a point of $S$ will be called an "ordinary" point and a point of $S^{\prime}$ which is not a point of $S$ will be called an "ideal" point. Regions in $S^{\prime}$ will be referred to as $G^{\prime}$-regions.

From here on the space to be considered is $S^{\prime}$.

THEOREM 63. In $S$, point and limit point are unchanged by the above definition of point and region in $S^{\prime}$.

THEOREM 64. Every point of $S^{\prime}$ is a limit point of $S$.

THEOREM 65. If $g^{\prime}$ is a $G^{\prime}$-region, then $\bar{g}^{\prime}=\overline{g^{\prime} \cdot S}$.

THEOREM 66. If $J$ is a simple closed curve of "ordinary" points, then $S^{\prime}-J$ is the sum of two mutually exclusive connected domains each having $J$ for its complete boundary. 
Proof. The point set $S-J$ is the sum of two mutually separate connected sets $H$ and $K$. Let $H^{\prime}=\bar{H}-\bar{H} \cdot J$ and $K^{\prime}=\bar{K}-\bar{K} \cdot J$. Suppose that $H^{\prime}$ has a point $A$ in common with $K^{\prime}$. The point $A$ is an "ideal" point $P_{[n q]}^{t}$. By Theorem 60 there exists a number $\bar{m}_{1}$ such that if $m>\bar{m}_{1}$ and $R_{m[n q]}^{t^{\prime \prime \prime}}$ belongs to $P_{[n q]}^{t}$, then $\bar{R}_{m[n q]}^{t^{\prime \prime \prime}} \cdot S$ contains no point of $J$. But $R_{m[n q]}^{t^{\prime \prime \prime}}$ contains points of both $H$ and $K$. Hence, $I_{m,[n q]}^{t^{\prime} t^{\prime \prime}}+\beta_{m,[n q]}^{t^{\prime \prime} t^{\prime \prime}}$ is a subset of $H$, say, and $I_{-m,[n(q+1)]}^{t^{\prime}{ }^{\prime \prime}}+\beta_{-m,[n(q+1)]}^{t^{\prime} t^{\prime \prime}}$ is a subset of $K$. By Theorem 61 there exists a number $\bar{m}_{2}$ such that if $m>\bar{m}_{2}$, then $U_{m[n q]}^{t}$ does not intersect $J$. But for each $m, U_{m[n q]}^{t}$ intersects both $\beta_{m,[n q]}^{t^{\prime} t^{\prime \prime}}$ and $\beta_{-m,[n(q+1)]}^{t^{\prime} t^{\prime \prime}}, t^{\prime \prime}<t<t^{\prime}$, which leads to a contradiction when $m$ is greater than both $\bar{m}_{1}$ and $\bar{m}_{2}$. Hence, $H^{\prime}$ and $K^{\prime}$ have no point in common. Since $H$ and $K$ are connected and have $J$ on their respective boundaries, $H^{\prime}$ and $K^{\prime}$ are connected and have $J$ for their respective boundaries. By Theorem $64, S^{\prime}=J+H^{\prime}+K^{\prime}$.

TheORem 67. If $P_{[n q]}^{l}$ belongs to the cluster of "ideal" points about the point $P_{j}$, and $g^{\prime}$ is a $G^{\prime}$-region containing the point $P_{[n q]}^{t}$ such that $g^{\prime} \cdot S$ is not a domain of $P_{[n q]}^{t}$, then $\bar{g}^{\prime} \cdot S$ contains $P_{j}$.

Proof. Suppose that $g^{\prime} \cdot S$ is not a domain of $P_{[n q]}^{t}$ but contains a domain $R$ of $P_{[n q]}^{t}$ and $\bar{g}^{\prime} \cdot S$ does not contain $P_{j}$.

Case I. If $g^{\prime} \cdot S$ is of type $\left(1^{\prime}\right), \dagger$ its boundary $J$ separates $R$ from $P_{j}$. Therefore, infinitely many of the simple closed curves $J_{1[n q]}, J_{2[n q]}, J_{3[n q]}, \cdots$ of $W_{j}$ would intersect $J$. But since $J$ is a compact subset of $S$, the sequential limit set of $J_{1[n q]}, J_{2[n q]}, J_{3[n q]}, \cdots$ of $W_{j}$ would contain points of $S$ other than $P_{j}$, contrary to Theorem 58 .

Case II. If $g^{\prime} \cdot S$ is of type $\left(2^{\prime}\right)$ and its boundary was used in the construction of the web $W_{j^{\prime}}$, then $j^{\prime}$ is not $j$ and one may see, from Theorem 58 and a method similar to that of Theorem 40 , that the boundary of $g^{\prime} \cdot S$ and a finite number of the arcs of the type $U_{m[n q]}^{t}$ of $W_{j^{\prime}}$ contains a simple closed curve $J$ lying in $S$ and separating $g^{\prime} \cdot S$, and hence $R$, from $P_{j}$. This leads to a contradiction as in Case I.

Case III. If $g^{\prime} \cdot S$ is of type $\left(3^{\prime}\right)$, there exists an integer $j^{\prime}$, such that $j^{\prime}$ is not $j$ and $g^{\prime} \cdot S$ was defined using the web $W_{j^{\prime}}$. Hence, $P_{j^{\prime}}$ is not $P_{j}$ and the boundary of $g^{\prime} \cdot S$ together with one of the simple closed curves of the type $J_{m[n q]}$ of $W_{j^{\prime}}$ contains a simple closed curve $J$ lying in $S$ and separating $g^{\prime} \cdot S$, and hence $R$, from $P_{j}$. This leads to a contradiction as in Case I.

Definition. For each $i$, let $G_{i}^{\prime}$ denote the collection of all $G^{\prime}$-regions $g^{\prime}$ of $S^{\prime}$ such that $D=g^{\prime} \cdot S$ is an element of $H_{i}$. The region $g^{\prime}$ is said to be defined by or obtained from $D$.

† See Notation and construction, p. 87. 
THEOREM 68. If $P_{[n q]}^{t}$ is an "ideal" point, there exists an integer $k$ such that no region of $G_{k}{ }^{\prime}$ contains $P_{[n q]}^{t}$ except those regions of $G_{k}{ }^{\prime}$ defined from domains belonging to $P_{[n q]}^{t}$.

Proof. Let $P_{\bar{j}}$ denote the edge point $P$ such that the cluster about $P$ contains $P_{|\mathrm{L} n|}^{t}$. Let $\bar{n}$ denote an integer such that $t>1 / \bar{n}$. There exists an integer $\bar{m}$ such that if $i$ is an integer greater than $\bar{m}$ and $R$ is a region in $S$ of $G_{i}$ of Axiom 1 which contains $P_{\bar{j}}$, then $R$ is a subset of $D_{\overline{n j}}$ in $S$. Suppose that $g^{\prime}$ is a region of $G_{i}^{\prime}, i>\bar{m}$, of the type $\left(1^{\prime}\right)$ or $\left(2^{\prime}\right)$ which contains $P_{[n q]}^{t} \cdot \dagger$ Then by Theorem $67, \bar{g}^{\prime} \cdot S$ contains $P_{\bar{j}}$. But $\bar{g}^{\prime} \cdot S$ is a subset of a region $R$ of $G_{1}$ of Axiom 1. Hence, $R$ contains $P_{\bar{j}}$ and points not belonging to $D_{\bar{n} j}$, which is a contradiction. Likewise, if $j \geqq \bar{m}$ and $j \neq \bar{j}$, no region of type $\left(3^{\prime}\right)$ obtained from a domain of an "ideal" point of the cluster about $P_{j}$ contains $P_{[n q]}^{t}$ because each such domain together with its boundary in $S$ is a subset of a region of $G_{i}$ of Axiom 1 with $i>\bar{m}$. Now by Theorem 58 , for each integer $j, j<\bar{m}$ and $j \neq \bar{j}$, there exists an integer $\bar{m}_{j}$ such that for $m>\bar{m}_{j}, \overline{\sum_{[n q]} I_{m[n q]}}$ defined from $W_{j}$ does not contain $P_{\bar{j}}$. Then if $g^{\prime}$ is a region of type $\left(3^{\prime}\right)$ of $G_{i}{ }^{\prime}, i>\bar{m}_{j}$, defined by $W_{j}, \bar{g}^{\prime} \cdot S$ does not contain $P_{\bar{j}}$. Hence, by Theorem 67, $R$ does not contain $P_{[n q]}^{t}$. Let $k$ denote an integer greater than $\bar{m}$ and for each $j, j<\bar{m}$ and $j \neq \bar{j}$, greater than $\bar{m}_{j}$. Thus, if $i>k$ and $g^{\prime}$ is a region of $G_{i}^{\prime}$ which contains $P_{[n q]}^{t}$, then $g^{\prime}$ was obtained from a domain of $P_{[n q]}^{t}$, for no two regions of type $\left(3^{\prime}\right)$ defined by $W_{\bar{j}}$ containing points of different spurs of the cluster about $P_{\bar{j}}$ have a point in common.

Theorem 69. The space $S^{\prime}$ satisfies Axiom 1.

Proof. It can be shown with the help of Theorems 67 and 68 that $S^{\prime}$ satisfies parts (1), (2), and (3) of Axiom 1.‡ Considerable argument is required but is left to the reader.

It will now be shown that part (4) of Axiom 1 is satisfied. Suppose that $M_{1}, M_{2}, M_{3}, \cdots$ is a sequence of closed subsets of $S^{\prime}$ such that for each $i$, $M_{i}$ contains $M_{i+1}$ and is a subset of a region $g_{i}^{\prime}$ of $G_{i}{ }^{\prime}$.

Case I. Suppose that for infinitely many values of $i, g_{i}{ }^{\prime}$ is of type $\left(1^{\prime}\right)$ or $\left(2^{\prime}\right)$. Suppose for simplicity that this is true for all integers $i$. Then for each $i, K_{i}=\left(\bar{g}_{1}{ }^{\prime} \cdot S\right) \cdot\left(\bar{g}_{2}{ }^{\prime} \cdot S\right) \cdots\left(\bar{g}_{i}{ }^{\prime} \cdot S\right)$ is a non-vacuous point set closed with respect to $S$ such that $K_{i}$ contains $K_{i+1}$. But for each $i, \bar{g}_{i}^{\prime} \cdot S$, and hence $K_{i}$, is a subset of a region $R_{i}$ of $G_{i}$ of Axiom 1. By Axiom 1 for $S$, there exists a point $P$ of $S$ which for each $i$ belongs to $K_{i}$. Hence in $S^{\prime}, P$ belongs to the

$\dagger$ See Notation and construction, p. 87.

$\ddagger$ See footnote p. 54. The role of the collections $G_{1}, G_{2}, G_{3}, \cdots$ is now to be taken by the collections $G_{1}^{\prime}, G_{2}^{\prime}, G_{3}^{\prime}, \cdots$. 
sequential limit set of $M_{1}, M_{2}, M_{3}, \cdots$ and therefore for each $i, P$ belongs to $M_{i}$.

Case II. If Case I does not occur, then there exists an integer $\bar{j}$ such that for infinitely many values of $i, g_{i}{ }^{\prime}$ is a $G^{\prime}$-region of type $\left(3^{\prime}\right)$ defined by the web $W_{\bar{j}}$; for otherwise infinitely many of these regions of type $\left(3^{\prime}\right)$ could be replaced by regions of type $\left(2^{\prime}\right)$ satisfying the above conditions. Suppose for simplicity that for each $i, g_{i}^{\prime}$ is of type $\left(3^{\prime}\right)$ and defined by $W_{\tilde{j}}$. Since no two regions of type $\left(3^{\prime}\right)$ defined by $W_{\bar{j}}$ which have a point in common contain points of different spurs of the cluster about $P_{\bar{j}}$, there exist for each $i$, integers $m, n$, and $q$, and numbers $t^{\prime}$ and $t^{\prime \prime}, 0<t^{\prime \prime}<t^{\prime}<1$, such that (1) $R_{m[n q]}^{t^{\prime} t^{\prime \prime}}$ defined from $W_{\bar{j}}$ is $g_{\imath}^{\prime} \cdot S$, (2) $[n q]$ does not vary with $i$, and (3) $m \rightarrow \infty$ and $\left(t^{\prime}-t^{\prime \prime}\right)$ $\rightarrow 0$, as $i \rightarrow \infty$. Since for each $i, g_{i}^{\prime}$ contains points of $g_{1}^{\prime}$, there exists a number $t, 0<t<1$, such that as $i \rightarrow \infty, t^{\prime} \rightarrow t$ and $t^{\prime \prime} \rightarrow t$. Hence $P_{[n q]}^{t}$ is common to $M_{1}, M_{2}, M_{3}, \cdots$.

THEOREM 70. Every $G^{\prime}$-region is a simple domain.

Theorem 71. The space $S^{\prime}$ satisfies Axioms $2,3,5_{1}^{*}$, and 7 and contains no edge points.

Theorem 71 is a consequence of Theorems 64,69 , and 70 and the fact that the collection of all $G^{\prime}$-regions is countable.

THEOREM 72. If $J$ is a simple closed curve of "ordinary" points, $I$ is one of its complementary domains, the points $A$ and $B$ separate the points $C$ and $D$ on $J$, and $A X B$ is an arc of "ordinary" points such that $\widehat{A X B}$ is a subset of $I$, then $I-\widehat{A X B}$ is the sum of two simple domains $I_{1}$ and $I_{2}$ such that the boundary of $I_{1}$ is the simple closed curve $A C B$ (of $\left.J\right)+A X B$ and the boundary of $I_{2}$ is the simple closed curve $A D B($ of $J)+A X B$.

Proof. Since the theorem holds for $S$, it can be shown to hold for $S^{\prime}$ with the help of Theorem 66.

THEOREM 73. Theorem 72 remains true when the segment $A X B$ contains just one "ideal" point.

Proof. Suppose for simplicity of notation that $X$ is the "ideal" point of the segment $A X B$. Let $W_{j}$ denote the web used in the definition of the domains of $X$, and let $N_{[n q]}$ denote the spur which contains $X$. A number of cases occur depending upon where the edge point $P_{j}$ is. Only one of these cases will be treated. The others may be dealt with in a somewhat similar but more complicated manner.

Suppose that $P_{j}$ does not belong to $J+A X B$. Let $J_{1[n q]}, J_{2[n q]}, J_{3[n q]}, \cdots$ denote the sequence of simple closed curves in $W_{j}$ which has as its sequential limit set $P_{j}+N_{[n q]}$. It can be shown by using Theorem 10 that there exists a 
sequence $C_{1}, C_{2}, C_{3}, \cdots$ of simple closed curves whose sequential limit set is $P_{j}+N_{[n q]}$ such that for each $k$, (1) there is an integer $m, m \geqq k$, so that $C_{k}$ is a subset of $J_{m[n q]}+A X B,(2) C_{k} \cdot \widehat{A X}$ is an $\operatorname{arc} A_{k}^{\prime} A_{k}^{\prime \prime}$ and $C_{k} \cdot \overparen{B X}$ is an $\operatorname{arc} B_{k}{ }^{\prime} B_{k}{ }^{\prime \prime}$, (3) the components of $C_{k}-C_{k} \cdot A X B$ are two segments, $A_{k}{ }^{\prime} X_{k} B_{k}{ }^{\prime}$ and $A_{k}^{\prime \prime} Y_{k} B_{k}^{\prime \prime}$, which approach the rays $\overrightarrow{A X}$ and $\overrightarrow{B X}$ in $X$ from different sides but $A_{k}^{\prime} X_{k} B_{k}^{\prime}$ and $A_{k+1}^{\prime} X_{k+1} B_{k+1}^{\prime}$ approach these rays from the same side, and (4) the common part of $C_{k}$ and $C_{k+1}$ is at most $P_{j}, C_{k}$ separating $C_{k+1}-C_{k+1} \cdot C_{k}$ from $J$. For each $k$, let $I_{1 k}$ and $I_{2 k}$ denote respectively the complementary domains of $A A_{k}^{\prime}$ (of $\left.A X\right)+A_{k}^{\prime} X_{k} B_{k}^{\prime}+B B_{k}^{\prime}$ (of $\left.B X\right)+A C B$ or $A A_{k}^{\prime \prime}$ (of $\left.A X\right)+A_{k}^{\prime \prime} Y_{k} B_{k}^{\prime \prime}+B B_{k}^{\prime \prime}$ (of $\left.B X\right)+A C B$ and $A A_{k}^{\prime}$ (of $A X$ ) $+A_{k}^{\prime} X_{k} B_{k}{ }^{\prime}+B B_{k}^{\prime}$ (of $\left.B X\right)+A D B$ or $A A_{k}^{\prime \prime}+A_{k}^{\prime \prime} Y_{k} B_{k}^{\prime \prime}+B B_{k}^{\prime \prime}$ (of $B X$ ) $+A D B$ which are subsets of $I$. Let $I_{1}$ denote $I_{11}+I_{12}+I_{13}+\cdots$ together with the limit points of this set which do not belong to $J+A X B$, and let $I_{2}$ denote $I_{21}+I_{22}+I_{23}+\cdots$ together with the limit points of this set which do not belong to $J+A X B$. These point sets, $I_{1}$ and $I_{2}$, can be shown to be domains satisfying the conclusions of Theorem 72 .

THEOREM 74. Theorem 72 remains true if the arc $A X B$ contains only a finite number of "ideal" points.

Proof. The method of proof will be that of mathematical induction. Theorem 73 shows that Theorem 74 holds true when the number of "ideal" points is one. We shall suppose that the theorem holds for $j-1$ or less points and show that it holds for $j$ points. Suppose that $A X B \cdot\left(S^{\prime}-S\right)=Z_{1}+Z_{2}+\cdots$ $+Z_{j}$. Let $N_{[n q]}$ denote a spur containing one of these "ideal" points, and let $Z_{r}$ denote that one of them on $N_{[n q]}$ which is farthest in $N_{[n q]}$ from its end point. Now the argument of Theorem 73 may be applied, $Z_{r}$ playing the role of $X$.

THEOREM 75. If $J$ is a simple closed curve containing only a finite number of "ideal" points, then $S^{\prime}-J$ is the sum of two mutually exclusive connected domains each having $J$ for its boundary.

Proof. Let $O$ denote an ordinary simple point of $J$, and let $R$ denote a simple domain whose boundary contains no "ideal" points such that $R \cdot J$ is a subset of an arc in $J$ of "ordinary" points. Let $A C B$ denote an arc of "ordinary" points in $R$ which has only the two points $A$ and $B$ in common with $J$ and such that an $\operatorname{arc} A D B$ of $J$ is a subset of $R$. It is now easy to see that, with the help of Theorem 74, Theorem 75 may be established.

THEOREM 76. Theorem 72 remains true regardless of the composition of the $\operatorname{arc} A X B$.

Proof. By slight modifications of the arguments of R. L. Moore a number 
of the theorems of Chapter III of Foundations can be shown to hold true in $S^{\prime}$ if the simple closed curves involved contain at most a finite number of "ideal" points. $\dagger$ In particular Theorem 13 of Chapter III of Foundations holds true in $S^{\prime}$ for simple closed curves which contain at most a finite number of "ideal" points. Theorem 76 may then be established in the following manner.

Since the boundaries of $G^{\prime}$-regions are simple closed curves containing at most a finite number of "ideal" points, it is easy to see that there exist two sequences of mutually exclusive arcs $A_{1}^{\prime} X_{1} B_{1}^{\prime}, A_{2}^{\prime} X_{2} B_{2}^{\prime}, A_{3}^{\prime} X_{3} B_{3}^{\prime}, \cdots$ and $A_{1}^{\prime \prime} Y_{1} B_{1}^{\prime \prime}, A_{2}^{\prime \prime} Y_{2} B_{2}^{\prime \prime}, A_{3}^{\prime \prime} Y_{3} B_{3}^{\prime \prime}, \cdots$ such that (1) for each $k$ the segments $A_{k}^{\prime} X_{k} B_{k}^{\prime}$ and $A_{k}^{\prime \prime} Y_{k} B_{k}^{\prime \prime}$ are subsets of $I$, contain at most a finite number of "ideal" points and the points $A_{k}^{\prime}, B_{k}^{\prime}, A_{k}^{\prime \prime}$, and $B_{k}^{\prime \prime}$ belong to segments $A C, B C, A D$, and $B D$ of $J$ respectively, (2) for each $k$, the complementary domain of $A_{k}^{\prime} X_{k} B_{k}^{\prime}+A_{k}^{\prime} A A_{k}^{\prime \prime}$ (of $\left.J\right)+B_{k}^{\prime} B B_{k}^{\prime \prime}$ (of $J$ ) $+A_{k}^{\prime \prime} Y_{k} B_{k}^{\prime \prime}$ which is a subset of $I$ contains the segment of $A X B$ and the corresponding complementary domain of $A_{k+1}^{\prime} X_{k+1} B_{k+1}^{\prime}+A_{k+1}^{\prime} A A_{k+1}^{\prime \prime}$ (of $\left.J\right)+B_{k+1}^{\prime} B B_{k+1}^{\prime \prime}$ (of $J$ ) $+A_{k+1}^{\prime \prime} Y_{k+1} B_{k+1}^{\prime \prime}$, and (3) the sequential limit set of either of these two sequences of $\operatorname{arcs}$ is $A X B$. If for each $k, I_{1 k}$ and $I_{2 k}$ denote respectively the complementary domains of $A_{k}^{\prime} C B_{k}^{\prime}$ (of $J$ ) $+A_{k}^{\prime} X B_{k}^{\prime}$ and $A_{k}^{\prime \prime} D B_{k}^{\prime \prime}$ (of $J$ ) $+A_{k}^{\prime \prime} Y B_{k}^{\prime \prime}$ which are subsets of $I$, then $I_{1}=\sum I_{1 k}$ and $I_{2}=\sum I_{2 k}$ satisfy the conclusions of Theorem 72 .

Now as in Theorem 75 it is possible to establish

THEOREM 77. If $J$ is a simple closed curve containing an arc of "ordinary" points, then $S^{\prime}-J$ is the sum of two mutually exclusive connected domains each having $J$ for its boundary.

With the help of Theorem 77 and methods similar to those in Foundations it can be shown that Theorem 74 remains true when the simple closed curve $J$ is of any nature, provided it contains an arc of "ordinary" points. With this in mind, it is easy to see that Theorem 76 remains true when $J$ is of any nature, provided it contains an arc of "ordinary" points. Then as in Theorem 74 it is possible to establish

TheOREM 78. If $J$ is a simple closed curve, $S^{\prime}-J$ is the sum of two mutually exclusive connected domains each having $J$ for its boundary.

Theorem 79. The space $S^{\prime}$ satisfies Axiom 4.

THEOREM 80. The space $S$ is homeomorphic with a subset of a space $S^{\prime}$ which satisfies Axioms 0-4, $5_{1}^{*}$ and 7 and contains no edge points.

$\dagger$ It may be helpful for the reader to note that an arc in $S^{\prime}$ may be approximated by an arc in $S^{\prime}$ which contains at most a finite number of "ideal" points since a $G^{\prime}$-region has at most two "ideal" points in its boundary. 
THEOREM 81. The space $S$ is homeomorphic with a subset of a plane or a sphere.

Proof. J. H. Roberts $\nmid$ has shown that $S^{\prime}$ of Theorem 80 is homeomorphic with a subset of a plane or a sphere.

Some of the preceding results will now be summarized for metric spaces

THEOREM 82. A locally connected, complete metric space in which the Jordan Curve Theorem $\ddagger$ is satisfied non-vacuously and in which Axiom $5_{1}^{*}$ holds true, is a cyclicly connected subset of a plane (if not compact) or a sphere (if compact).

Proof. A locally connected complete metric space satisfies Axioms 0, 1, and 2. And such a space in which Axiom $5_{1}^{*}$ holds true satisfies Axioms 4 and 3 if the Jordan Curve Theorem holds true non-vacuously (Theorem B). Axiom 7 holds true in such a space by Theorem 31. Thus Theorem 82 follows from Theorem 81.

TheOREM 83. A locally connected, locally peripherally connected, complete metric space in which the Jordan Curve Theorem is non-vacuously satisfied is a cyclicly connected subset of a plane or a sphere.\$

Theorem 84. A locally connected, locally peripherally compact, complete metric space in which the Jordan Curve Theorem is non-vacuously satisfied is a cyclicly connected subset of a plane or a sphere.

$\dagger$ J. H. Roberts, Concerning compact continua in certain spaces of $R$. L. Moore, Bulletin of the American Mathematical Society, vol. 39 (1933), pp. 615-621.

$\ddagger$ Axiom 4 .

$\S$ See p. 53.

The UNIVERSity OF TeXas, Austin, Texas 